\title{
POINT SETS AND ALLIED CREMONA GROUPS*
}

\author{
(PART III)
}

BY

\author{
ARTHUR B. COBLE†
}

\section{INTRODUCTION}

In Part I $\ddagger$ of this series projectively distinct sets $P_{n}^{k}$ of $n$ points in $S_{k}$ were mapped upon points of a space $\Sigma_{k(n-k-2)}$ and a certain Cremona group $G_{n !}$ in $\Sigma$ was induced by permutation of the points of the set. For $P_{5}^{2}$ and $P_{6}^{2}$ these groups furnished an effective algebraic background for exhibiting solutions of the quintic and sextic equations. In Part II§ the $G_{n !}$ appeared as merely a subgroup of a more important group $G_{n, k}$ in $\Sigma_{k(n-k-2)}$ which also is defined by $P_{n}^{k}$. In particular the $G_{6 !}$ in $\Sigma_{4}$ attached to $P_{6}^{2}$ is a subgroup of the $G_{6,2}$ in $\Sigma_{4}$, which has the order 51840 and is isomorphic with the group of the lines on a cubic surface.

It is the purpose of this. Part III to show first that the lines of a given cubic surface can be determined rationally in terms of a solution of the form problem of $G_{6,2}$; and second that this solution can be obtained in terms of the solution of a form problem which arises in connection with the theta functions. $\|$ The presentation follows a line quite different from that suggested by Klein. A striking difference is that we make no actual or implied use of an equation of degree 27 or other resolvent equation. All of the operations required are effected within the domain of irrational invariants and covariants of the

* Presented in part to the Society, August 3, 1915.

$\dagger$ This investigation has been carried out under the auspices of the Carnegie Institution of Washington, D. C.

$\ddagger$ These Trans a ctions, vol. 16 (1915).

$\S$ These Transactions, vol. 17 (1916).

|| That an equation of degree 27 for the lines of a cubic surface could be solved by hyperelliptic modular functions was first pointed out by Klein in a letter to Hermite, J o u r n a l de mathématiques, ser. 4, vol. 4 (1888), p. 169 . His suggestions were elaborated by Witting, M a the mat is che A n n a le n, vol. 29 (1887), p. 167; by Maschke, ibid., vol. 33 (1889), p. 317; and by Burkhardt, Grundzüge einer allgemeinen Systematik der hyperelliptischen Functionen I. Ordnung, ibid., vol. 35 (1890), p. 198, vol. 38 (1891), p. 161, and vol. 41 (1893), p. 313. The latter articles are referred to as BI, BII, and BIII. Since Maschke and Burkhardt respectively have determined complete systems for the so-called "group of the $Z$ 's " and "group of the Y's" we shall indicate these groups and the associated form problems by attaching their names. 
surface-a domain defined by the adjunction of all the lines considered as comitants of the surface.* Again the method of Klein is based primarily on the existence of the Maschke group and its derived group of line transformations while $G_{6,2}$ leads more naturally to the Burkhardt group. $\dagger$ We are thus enabled to dispense with a separation of the roots of the sextic underlying the theta functions of genus two. Finally by developing a covariant form for the normal hyperelliptic surface certain interesting geometric facts, pertaining to the collineation groups in question, are obtained upon which the solution of their form problems is based. $\ddagger$

In $\S 1$ the $G_{6,2}$, its generators, invariants, and form problem are discussed, while in $\S 2$ it is shown that the adjunction of a solution of this form problem serves to determine the lines of a given cubic surface. In $\S 3$ the simplest linear system of irrational invariants of the surface is set forth. Under permutation of the lines the members of this system are transformed under the operations of a correlation group which is built up on the Burkhardt collineation group. The form problem of $G_{6,2}$ is solved in $\S 4$ by the adjunction of a solution of the Burkhardt form problem. The facts obtained in $\S 5$ concerning the hyperelliptic surface serve in $\S 6$ as a basis for the solution of the special§ Burkhardt form problem in terms of hyperelliptic modular functions. Finally in $\S 7$ the general Burkhardt form problem is solved in terms of the special problem. The comparison of this determination of the lines of a cubic surface with the solution of a quintic equation detailed in $\S 8$ reveals a remarkable analogy between these two problems-an analogy which furnishes perhaps the best evidence of the value of the methods here employed.

\section{The EXtended GRoup $G_{6,2}$}

For a given ordered set of 6 points in a plane we choose as in Part $I$ the reference scheme and factors of proportionality so that the coördinates of the points take the form
(1) $1,0,0$,
(4) $1,1,1$,
(2) $0,1,0$,
(5) $x, y, u$,
(3) $0,0,1$,
(6) $z, t, u$.

The $P_{6}^{2}$ is then represented by the point $P$ in $\Sigma_{4}$ with coördinates $x, y, z, t, u$.

* This removes the objection-noted by Burkhardt (BIII, p. 342, 3)-that while the lines are in rational relation they are not in covariant relation to the roots of such an equation. Equations whose roots are irrational invariants of the surface can be formed but the degree of these invariants is too high for practical discussion. below.

† Since no solution of the Burkhardt form problem has been given hitherto this is supplied

$\ddagger$ The results here obtained indicate some errors in the formulas of Burkhardt; cf. footnotes $\S \S 4,5,6$.

$\S$ I. e., the Burkhardt form problem for which $J_{4}=0$. 
As the points of $P_{B}^{2}$ are permuted and the points of the re-ordered set transformed again into the form (1) the point $P$ is transformed into its conjugate positions under $G_{6 !}$. A set of generators $I_{12}, \cdots, I_{56}$ of $G_{6 !}$ is given in (2) below where $I_{i k}$ is the involutory operation of $G_{6 !}$ which arises from the intarchange of the points $p_{i}$ and $p_{k}$ of $P_{6}^{2}$. A quadratic transformation $I_{123}$ with fundamental points ( $F$-points) at $p_{1}, p_{2}, p_{3}$ and corresponding inverse $F$ points at $p_{1}^{\prime}, p_{2}^{\prime}, p_{3}^{\prime}$ transforms $p_{4}, p_{5}, p_{6}$ into $p_{4}^{\prime}, p_{5}^{\prime}, p_{6}^{\prime}$. Then by definition the sets $P_{6}^{2}$ and $P_{6}^{\prime 2}$ are congruent under $I_{123}$. If the set ${P_{6}^{\prime 2}}^{2}$ be projected into the form (1) the coördinates of its map $P^{\prime}$ in $\Sigma_{4}$ in terms of those of $P$ are (II, $\S 3$ ) as given in the table:

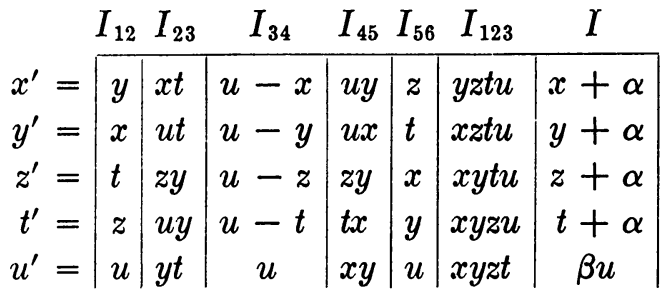

$$
\begin{aligned}
& \alpha=\frac{z y(x+t-u)-x t(y+z-u)}{x t-y z-u(x+t-y-z)}, \quad \beta=1+\alpha \frac{x+t-y-z}{x t-y z} .
\end{aligned}
$$

Here $I$ (the element B of I, $\S 10$ ) is the involutory transformation determined by associated sets $P_{6}^{2}$ and $P_{6}^{\prime 2}$.

We have seen (II, $\S 3$, p. 358) that the $G_{6 !}$ generated by $I_{12}, \cdots, I_{56}$ together with the $I_{123}$ generate a group $G_{6,2}$ in $\Sigma_{4}$ of order 51840; and further that the 15 involutions $I_{i k}$, the 20 involutions $I_{i j k}$, and the involution $I$ constitute a set of 36 conjugate generators of $G_{6,2}$. Those elements of $G_{6,2}$ which can be expressed as a product of an even number of generators lie in the invariant subgroup $\Gamma_{6,2}$ (II, $\S 7(51)$ ) of order 25920 . To a set of 51840 conjugate points under $G_{6,2}$ in $\Sigma_{4}$ there corresponds in the plane a set of 51840 projectively distinct ordered $P_{6}^{2}$ 's such that any one of the $P_{6}^{2}$ 's is congruent in some order under ternary Cremona transformation to any other of the $P_{6}^{2}$ 's. Disregarding the 6 ! possible orders we have then just 72 projectively distinct $P_{6}^{2}$ 's. The $72 P_{6}^{2}$ 's divide under $I$ into 36 pairs of associated $P_{6}^{2}$ 's. The original $P_{6}^{2}$ and its associated $P_{6}^{2}$ determine the group $G_{6 ! \cdot 2}$ of I, $\S 10$. The property of congruent $P_{6}^{2}$ 's most important for our purpose is

(3) If the system of cubic curves on $P_{6}^{2}$ can be transformed by ternary Cremona transformation into the system of cubic curves on $P_{6}^{\prime 2}$ then the maps of $P_{6}^{2}$ and ${P_{6}^{\prime}}^{2}$ in $\Sigma_{4}$ are conjugate points under $G_{6,2}$.

According to I, $\S 4$, we can choose six cubic curves $a, \cdots, f$ on $P_{6}^{2}$ such that

$$
\begin{aligned}
a+\cdots+f & \equiv 0, \\
\bar{a} a+\cdots+\overline{f f} & \equiv 0, \\
\bar{a}+\cdots+\bar{f} & =0 .
\end{aligned}
$$


Explicit expressions for $a, \cdots, f, \bar{a}, \cdots, \bar{f}$ in terms of the coördinates of $P_{6}^{2}$ are given there. The plane is thus mapped upon a cubic surface $C^{(3)}$ whose equation is

$$
C^{(3)} \equiv a^{3}+\cdots+f^{3}=0 .
$$

The equations of the 45 tritangent planes (and thereby also the equations of the 27 lines) of $C^{(3)}$ also are explicitly given in terms of $P_{6}^{2}$. The above hexahedral form of $C^{(3)}$ is associated with a double six of lines on $C^{(3)}$ and precisely that double six mapped from the six points $p_{i}$ of $P_{6}^{2}$ and the six conics on points other than $p_{i}(i=1, \cdots, 6)$.

The invariants of the hexahedral $C^{(3)}$ are symmetric polynomials in $\bar{a}$, $\cdots, \bar{f}$ which are unaltered to within a factor when $\bar{a}, \cdots, \bar{f}$ are replaced by the $\bar{a}^{\prime}, \cdots, \bar{f}^{\prime}$ attached to any other hexahedral form of $C^{(3)}$. The complete system of a cubic surface in $S_{3}$ according to Salmon and Clebsch* contains invariants $i_{8}, i_{16}, i_{24}, i_{32}$, and $i_{100}$ of the degrees indicated and of weights $6,12,18,24,30$, and 75 respectively. The hexahedral surface appears as a section in $S_{5}$ (variables $a, \cdots, f$ ) of a cubic spread with numerical coördinates. The Clebsch transference principle states that the invariants of the section are obtained by bordering the symbolic determinants of the invariant in $S_{3}$ by the coördinates of the spaces of section and by applying the determinants to the spread in $S_{5}$. Since the coefficients of the spread in $S_{5}$ are merely numerical each unit of weight contributes a unit to the degree in $\bar{a}, \cdots, \bar{f}$, and the invariants of the hexahedral form are $I_{6}, I_{12}, I_{18}, I_{24}, I_{30}$, and $I_{75}$ of the degrees indicated in $\bar{a}, \cdots, \bar{f}$. When $\bar{a}, \cdots, \bar{f}$ are expressed in terms of the $x, y, z, t, u$ of $P_{6}^{2}$ or of the point $P$ in $\Sigma_{4}$ the process of expressing an invariant $I$ in terms of the $\bar{a}^{\prime}, \ldots, \bar{f}^{\prime}$ corresponds to the process of replacing $P_{6}^{2}$ by a congruent $P_{6}^{\prime 2}$, or in $\Sigma_{4}$ to the process of passing to a conjugate point $P^{\prime}$ under $G_{6,2}$. Since the relation of $I$ to $C^{(3)}$ is unaltered by this operation, the corresponding expression for $I$ in terms of $x, y, z, t, u$ must be unaltered to within a factor by the operations of $G_{6,2}$. Hence to the invariants $I$ of weight $w$ of $C^{(3)}$ there correspond invariant spreads $I$ of $G_{6,2}$ in $\Sigma_{4}$ determined to within a power $\lambda^{w}$ of an indeterminate factor of proportionality $\lambda$. That but one such factor $\lambda$ can occur is due to the fact that linear systems of invariants can be formed as soon as two invariants appear. Here the invariant $I_{75}$ may be an exception since it cannot lie in a linear system determined by itself and earlier invariants because of its odd degree. This ambiguity in the factor $\lambda$ can however extend only to the sign since $I_{75}^{2}$ is no longer exceptional. Conversely every invariant $I$ of $G_{6,2}$ must arise in this way from some invariant of $C^{(3)}$. By an invariant $I$ of $G_{6,2}$ we mean a form in $x, y, z, t, u$ which is reproduced to within a factor by any operation of $G_{6,2}$. For the coördinates $x, y, z, t, u$ of $P$ are themselves irrational invariants of

\footnotetext{
* Cf. Pascal; Repertorium I, p. 342 (Teubner, 1900).
} 
$C^{(3) *}$ in the projective sense which are rational in the domain obtained by the separation of the lines. The given form is therefore a projective invariant in this domain and, being invariant under the operations of $G_{6,2}$, it must be independent of admissible permutations of the lines and therefore must be rational in the coefficients of $C^{(3)}$. According to I, p. 196, the $\bar{a}, \cdots, \bar{f}$ are of degree two in $x, y, z, t, u$ and we shall find $\dagger$ that for $w=6 k$ the factor $u^{2 k}$ can be removed from each invariant of weight $w$, whence

(6) To the complete system of invariants $i_{8}, \cdots, i_{32}, i_{40}, i_{100}$ of the general $C^{(3)}$ there corresponds the complete system $I_{6}, \cdots, I_{24}, I_{30}, I_{75}$ of the hexahedral $C^{(3)}$, and the complete system $I_{5.2}, \cdots, I_{5 \cdot 8}, I_{5 \cdot 10}, I_{5 \cdot 25}$ of spreads of the orders indicated invariant under $G_{6,2}$ in $\Sigma_{4}$.

The equation problem determined by the $G_{6,2}$ can now be formulated as follows:

(7) Given numerical values of $I_{5 \cdot 2}, I_{5.4}, \cdots, I_{5 \cdot 10}$ to calculate the ratios of the coördinates $x, y, z, t, u$ of a point $P$ in $\Sigma_{4}$ for which the given spreads can take the assigned values.

From one solution of this problem there can be obtained by the operations of $G_{6,2}$ a set of 51840 solutions. It is not easy to prove directly that no other solutions exist, since these spreads have common manifolds determined by the reference basis in $\Sigma$. The fact can be inferred later from the number of solutions of the Burkhardt form problem.

It had been noted by Klein (loc. cit.) that the adjunction of the square root of the discriminant $\Delta$ of $C^{(3)}$ reduced the order of the group of the lines to 25920 . Evidently $\Delta$ is the most convenient fourth invariant and we shall suppose hereafter that $i_{32}, I_{24}$, or $I_{5.8}$, as the case may be, is $\Delta$. The $\sqrt{\Delta}$ can be calculated explicitly in terms of the $\bar{a}, \cdots, \bar{f}$ without much trouble. The surface when mapped by means of $P_{6}^{2}$ has a double point when either six points are on a conic, or three points are on a line, or two points coincide in some direction. Thus $\Delta$ has $1+20+15=36$ irrational factors which will be indicated respectively by $\delta, \delta_{i j k}, \delta_{i j}$. Recalling the notation of I, pp. 170-3, we have $\delta=d_{2}$ and $\delta_{123} \delta_{456}=(\bar{a}+\bar{b}+\bar{c})=-(\bar{d}+\bar{e}+\bar{f})$, etc. In order that a rational expression in $\bar{a}, \cdots, \bar{f}$ may be obtained we must use $d_{2}^{2}=a_{2}^{2}-4 a_{4}$ and therefore must use the squares of the factors $\delta_{i j k}$ as well. This raises the product of $\delta^{2}$ and the ten squares $\delta_{i j k}^{2}$ to the proper degree, 24 , of $\Delta$ in $\bar{a}, \cdots, \bar{f}$ so that the factors $\delta_{i j}$ do not occur explicitly. This is to be expected since coincidence in a given direction cannot be expressed by a single condition on the coördinates of $P_{6}^{2}$. A definite sign can be given to $\sqrt{\Delta}$ by the assumption

$$
\sqrt{\Delta}=d_{2} \prod_{10}(\bar{a}+i+j),
$$

* E. g., if $x=0$, points $p_{2}, p_{3}, p_{5}$ are collinear and $C^{(3)}$ has a node.

$\dagger$ Cf. $\S 3$. 
where the sign of $d_{2}$ is defined in I, $\S 4(47)$, and where $i, j$ run over the 10 pairs drawn from $\bar{b}, \cdots, \bar{f}$. The product $\Pi$ can be conveniently evaluated by symmetrizing successively for $3,4, \cdots, 6$ letters. If in terms of $a_{2}$, $\cdots, a_{6}$, the elementary symmetric functions of $\bar{a}, \cdots, \bar{f}$, we set

$$
\begin{gathered}
q_{4}=a_{2}^{2}-4 a_{4}, \quad q_{5}=a_{2} a_{3}-2 a_{5}, \\
I_{6}{ }^{*}=3 a_{3}^{2}-4 a_{2} q_{4}-12 a_{6},
\end{gathered}
$$

then $\sqrt{\Delta}$ takes the form

$$
\sqrt{\Delta}=d_{2}\left(q_{4} I_{6}+4 a_{2} q_{4}^{2}-3 q_{5}^{2}\right) .
$$

We have seen (I, p. 196) that $d_{2}$ changes sign under the involution $I_{12}$ while $\bar{a}, \cdots, \bar{f}$ and therefore $a_{2}, \cdots, a_{6}, q_{4}, q_{5}, I_{6}$ do not; whence $\sqrt{\Delta}$ changes sign under the operations of $G_{6,2}$ not contained in $\Gamma_{6,2}$ and is invariant under $\Gamma_{6,2}$.

The skew invariant, $i_{100}$ or $I_{75}$, has the same behavior under $G_{6,2}$ and $\Gamma_{6,2}$ as $\sqrt{\Delta}$. This invariant has 45 irrational factors. For if we recall from I, p. 197, that $\bar{a}-\bar{d}=0$ is the condition that the lines $\overline{12}, \overline{34}, \overline{56}$ in $S_{2}$ meet in a point we find 15 similar irrational invariants whose product is there denoted by $\sqrt{d}$. If then we carry out on $\bar{a}-\bar{d}=0$ the involution $I_{123}$ it must be transformed into the condition that the conic on $p_{1}, p_{2}, p_{5}, p_{6}$ is touched at $p_{3}$ by the line $\overline{34}$. This condition is

$$
j_{1256,3} \equiv\left|\begin{array}{llll}
\overline{126} & \overline{356} & \overline{136} & \overline{256} \\
\overline{123} & \overline{354} & \overline{134} & \overline{253}
\end{array}\right|=0 .
$$

Thus $j_{1256,3}$ is of degree $2,2,3,1,2,2$ in the points in order. The product of the 30 irrational invariants of this type is of degree 60 in each of the six points; let us call it $f_{60}$. It is easy to verify that, while $j_{1256,3}$ is changed in sign by $I_{12}$, yet $f_{60}$ is unaltered. Since $f_{60}$ does not contain the factor $d_{2}$ it must (cf. I (49)) be a rational integral function of $a_{2}, \cdots, a_{6}$. Hence

$$
I_{75}=\sqrt{d} \cdot f_{60}
$$

is changed in sign along with $\sqrt{d}$ by the involution $I_{12}$ and therefore is an exception to the rule that invariants of $C^{(3)}$ are invariants under $G_{6,2}$.

The above identification of $\sqrt{d} \cdot f_{60}$ with $I_{75}$ which is indicated by their degrees can be substantiated by their geometric interpretation. The vanishing of $I_{75}$ is the condition that $C^{(3)}$ be unaltered by an involutory collineation. On the other hand if $\bar{a}-\bar{d}=0$ then (cf. I (16)) $P_{6}^{2}$ is self-associated in the order (12) (34) (56), the net of cubics on $P_{6}^{2}$ is transformed into itself by the corresponding Cremona involution of order 5 , and the cubic surface admits an involutory collineation.

(13) A complete system of invariants for $\Gamma_{6,2}$ consists of $I_{6}, I_{12}, \sqrt{\Delta}, I_{18}$, $I_{30}$, and $I_{75}$.

* We shall verify later ( $\$ 3(38))$ that this is the first invariant of $C^{(3)}$. 
The degrees of all the invariants of $\Gamma_{6,2}$ which we shall have occasion to use hereafter are such that $I_{75}$ cannot occur in their expressions. The form problem for the group $\Gamma_{6,2}$ reads as follows:

(14) Given the numerical values of $I_{5.2}, I_{5 \cdot 4}, \sqrt{\Delta}, I_{5 \cdot 6}$, and $I_{5.10}$ to calculate the ratios of the coördinates of a point $P$ in $\Sigma_{4}$ for which these spreads can take the assigned values.

Clearly the adjunction of $\sqrt{\Delta}$ suffices to reduce the group $G_{6,2}$ to the group $\Gamma_{6,2}$. For the linear system $\lambda I_{6}^{2}+\mu I_{12}+\nu \sqrt{\Delta}$ is invariant under $\Gamma_{6,2}$ but not under $G_{6,2}$. Hence if the form problem of $G_{6,2}$ has 51840 solutions, that of $\Gamma_{6,2}$ has 25920 solutions, and vice versa.

\section{Determination of the lines of a cubic surface in terms of the FORM PROBLEM OF $G_{6,2}$}

If $(c x)^{3}$ is a general quaternary cubic form the cubic surface $C^{(3)}$ is $(c x)^{3}=0$. We seek the 27 sets of six line coördinates $\pi_{i k}$ determined by any pair of planes on each of the 27 lines of $C^{(3)}$. Such pairs can be selected from the 45 tritangent planes of $C^{(3)}$ so that we shall merely need expressions $\left(t_{i} x\right)=0$ $(i=1, \cdots, 45)$ for these planes.

We shall assume first that for the given form $(c x)^{3}$ a series of covariant processes has been outlined, following some one of the known complete systems of the cubic surface, which will furnish definite values for the invariants $i_{8}, i_{16}, i_{24}, i_{32}$, and $i_{40}$ of $C^{(3)}$ and definite expressions, $\left(l_{11} x\right),\left(l_{19} x\right),\left(l_{27} x\right)$, and $\left(l_{43} x\right)$ for the linear covariants of $C^{(3)}$.

In any one of 36 ways the given form $(c x)^{3}$ can be expressed in Cremona's hexahedral form (4). We shall assume second that through the use of the Clebsch transference principle which does not affect covariant relations, the same series of covariant processes has been carried out on the Cremona form and that there has been obtained explicit expressions in terms of $\bar{a}, \cdots, \bar{f}$ of the corresponding invariants and linear covariants, viz: $I_{6}, I_{12}, I_{18}, I_{24}$, $I_{30}$, and $\left(L_{8} a\right),\left(L_{14} a\right),\left(L_{20} a\right),\left(L_{32} a\right)$ of the degrees in $\bar{a}, \cdots, \bar{f}$ indicated by the subscripts. The two investigations here assumed have of course a wider range of application than we shall need. The first is in quite satisfactory shape. The second has been begun* and if completed would afford a method for settling all questions concerning the relation of the lines and tritangent planes of $C^{(3)}$ to the covariant system of $C^{(3)}$.

If the coefficients of $(c x)^{3}$ and $\bar{a}, \cdots, \bar{f}$ be known then the linear transformation

$$
\begin{aligned}
\left(l_{11} x\right) & =\left(L_{8} a\right), & \left(l_{19} x\right) & =\left(L_{14} a\right), \\
\left(l_{27} x\right) & =\left(L_{20} a\right), & \left(l_{43} x\right) & =\left(L_{32} a\right), \\
0 & =(\bar{a} a), & 0 & =(a),
\end{aligned}
$$

* Sousley, American Journal of Mathematics, vol. 38 (1916). 
furnishes the typical representation of the given cubic surface in the hexahedral form. The determinant of the four linear forms in $x$ of (15) is $i_{100}$; of the six linear forms in $\bar{a}, \cdots, \bar{f}$ is $I_{75}$.

The processes involved in the determination of the 45 tritangent planes of the given cubic surface $C^{(3)}$ can now be outlined as follows:

$1^{\circ}$. For the given $C^{(3)} \equiv(c x)^{3}=0$, the invariants $i_{8}, \cdots, i_{40}$ and the linear covariants $\left(l_{11} x\right), \cdots,\left(l_{43} x\right)$ are calculated.

$2^{\circ}$. The values of $i_{8}, \cdots, i_{40}$ furnish the values of the known quantities $I_{6}, \cdots, I_{30}$ of the equation problem of $G_{6,2}$. We assume that this problem has been solved and that therefore the coördinates $x, y, z, t, u$ of a point $P$ in $\Sigma_{4}$ are known.

$3^{\circ}$. These coördinates substituted in (1) furnish a set $P_{6}^{2}$ by means of which the given $C^{(3)}$ can be mapped upon $S_{2}$. The values of $\bar{a}, \cdots, \bar{f}$ and $d_{2}$ in terms of these coördinates are given in I (35), (47).

$4^{\circ}$. From the values of $\bar{a}, \cdots, \bar{f}$ and $d_{2}$ the linear covariants $\left(L_{8} a\right), \cdots$, $\left(L_{32} a\right)$ of (15), and the 45 tritangent planes as given in I (47) are expressed linearly in terms of $a, \cdots, f$.

$5^{\circ}$. From equations (15) the values of $a, \cdots, f$ as linear functions of $x$ are obtained.

$6^{\circ}$. These values of $a, \cdots, f$ set in the equations of the 45 tritangent planes furnish the equations of the tritangent planes of the given surface $(c x)^{3}=0$ in terms of the given variables $x$.

The above procedure is effective whenever the equations (15) are such that step $5^{\circ}$ can be carried out. We have therefore a case of exception when $i_{100}=I_{75}=0$. In this case however the Galois group of the problem is reduced at least to a desmic group of order 2.576 and the lines of $C^{(3)}$ can be expressed by means of radicals alone.

Since any solution of the equation problem of $\Gamma_{6,2}$ is equally well a solution of that of $G_{6,2}$, all the operations outlined above can be carried out after the adjunction of $\sqrt{\Delta}$ has reduced the group of the problem to $\Gamma_{6,2}$ of order 25920. We are now enabled to dispense with the originally given surface $C^{(3)}$ and to consider further only those processes which are necessary for the solution of the equation problem of $\Gamma_{6,2}$.

\section{IRrational invariants of $C^{(3)}$}

In this paragraph certain systems of irrational invariants of $C^{(3)}$ are built up from the irrational factors of its discriminant. They correspond to the separation of the lines of the surface into double sixes, into the so-called " complexes" of lines, and into the triads of lines in a tritangent plane. Of these three sorts the first do not give rise to irrational invariants of $P_{6}^{2}$ as defined in I, $\S 3$, for their expressions in terms of the coördinates of $P_{6}^{2}$ are not 
homogeneous and of the same degree in the coördinates of each point. But the last two sorts are irrational invariants of $P_{6}^{2}$ and they lie in the simplest linear system of spreads invariant under the extended group $G_{6,2}$ of $P_{6}^{2}$. The operations of $G_{6,2}$ when carried out on this linear system give rise to a collineation group of order 51840 which we shall identify with a group of correlations in $S_{4}$ which has the Burkhardt group for its collineation subgroup.

Let us denote the lines of $C^{(3)}$ by $l_{1}, \ldots, l_{6} ; m_{1}, \cdots, m_{6} ; l_{12}, \cdots, l_{56}$ according as they correspond in the plane of $P_{6}^{2}$ to respectively the directions about the points $p_{1}, \cdots, p_{6}$; the conics on the six points other than $p_{1}, \cdots, p_{6}$; and the lines

$$
(12 x)=0, \quad \cdots, \quad(56 x)=0 .
$$

In the above notation a double six is isolated and the 36 double sixes divide into three sets

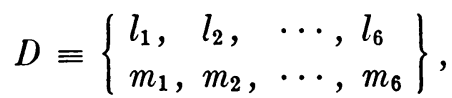

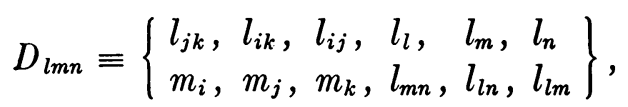

$$
\begin{aligned}
& D_{\imath j} \equiv\left\{\begin{array}{ll}
l_{i}, m_{i}, l_{j k}, l_{j l}, l_{j m}, l_{j n} \\
l_{j}, m_{j}, l_{i k}, l_{i l}, l_{2 m}, l_{i n}
\end{array}\right\}, \\
& (i, j, k, \cdots, n=1,2, \cdots, 6) .
\end{aligned}
$$

In the plane, $D_{l m n}$ arises from $D$ by using the quadratic transformation $A_{i j k}$; while $D_{i j}$ arises from $D$ by using the cubic transformation with double $F$ point at $p_{j}$ and simple $F$-points at $p_{k}, \cdots, p_{n}$. The double six $D$ has 6 lines in common with each of the 20 double sixes $D_{l m n}$ and 4 lines in common with each of the 15 double sixes $D_{i j}$. Two double sixes form an azygetic or syzygetic duad according as they have 6 or 4 lines in common. The typical azygetic duads are:

$$
D, D_{i j k} ; \quad D_{i j k}, D_{l m n} ; \quad D_{i j k}, D_{i j l} ; \quad D_{i j k}, D_{i l} ; \quad D_{i j}, D_{i k} ;
$$

the typical syzygetic duads are:

$$
D, D_{i j} ; \quad D_{i j k}, D_{i l m} ; \quad D_{i j k}, D_{l m} ; \quad D_{i j k}, D_{i j} ; \quad D_{i j} ; D_{k l} . *
$$

* The terms azygetic and syzygetic are taken from the finite geometry mod $2(p=3)$ of the theta functions (cf. II, p. 357). If of the $28 O$ quadrics one, say $Q_{07}$, be isolated then the 36 points $P_{07}, P_{0 i j k}=P_{l m r 7}, P_{i j}$ which are not on this quadric can be associated with the double sixes while the 27 points $P_{0 i}, P_{7 i}$, and $P_{i j 07}$ which are on the quadric can be associated with the 27 lines of $C^{(3)}$. Through each point outside the quadric there pass 6 secants of the quadric. These are ordinary lines of the null system determined by the quadric. Two points are syzygetic or azygetic according as they do or do not lie on a null line. Thus each outside point (or double six) determines 6 pairs of points on $Q_{07}$ (line pair of $C^{(3)}$ ) and these 6 pairs lie in two sets of 6 for each point is azygetic with all of its own set and one of the other set. Any two outside points may be syzygetic or azygetic. In the first case their join touches the quadric at a point; in the second case their join is skew to the quadric and the three points on it correspond to an azygetic triad of double sixes. 
Two azygetic double sixes determine a third such that any two of the three are azygetic. There are 120 such azygetic triads whose typical forms are:

$$
D, D_{i j k}, D_{l m n} ; \quad D_{i j k}, D_{i j l}, D_{k l} ; \quad D_{\imath j}, D_{i k}, D_{j k} \text {. }
$$

No line is common to the three double sixes of an azygetic triad so that the triad contains 18 lines each appearing in two double sixes and omits 9 lines.

The double sixes which are syzygetic with the members of an azygetic triad lie in two other azygetic triads such that double sixes from any two of the triads are syzygetic. Such a symmetrical set of three azygetic triads is a complex. The 9 lines omitted from each triad of a complex make up the 27 lines. There are 40 such complexes; 10 of the form

$$
\Gamma_{i j k, l m n} \equiv D, D_{i j k}, D_{l m n} ; \quad D_{i j}, D_{i k}, D_{j k} ; \quad D_{l m}, D_{l n}, D_{m n} ;
$$

and 30 of the form

$$
\Gamma_{i j, k l, m n} \equiv D_{i j}, D_{i k l}, D_{j k l} ; \quad D_{k l}, D_{k m n}, D_{l m n} ; \quad D_{m n}, D_{m i j}, D_{n i j} .
$$

The last type depends upon the separation of $i, \cdots, n$ into three pairs and upon the cyclic arrangement of the pairs.*

We have noted in $\S 1$ that there are 36 particular types of sets $P_{6}^{2}$ for which $C^{(3)}$ has a double point, each type being associated with a double six. The six lines of half the isolated double six map into the six lines of $C^{(3)}$ on the double point and the six lines of the other half coincide and map into directions at the double point. The 36 corresponding irrational factors of the discriminant $\Delta$ of $C^{(3)}$ have been denoted by $\delta, \delta_{l m n}, \delta_{i j}$. The vanishing of each of these factors can be expressed by explicit conditions on the coördinates of $P_{6}^{2}$ except for the factors of type $\delta_{i j}$ which indicate a coincidence of the points $p_{i}$ and $p_{j} \cdot \dagger$ We shall now set forth a set of 40 irrational invariants of $C^{(3)}$ which correspond to the 40 complexes defined above.

If we consider the product

$$
\gamma_{123,456}=d_{2} \cdot(123)(456)
$$

we see that it is of degree 3 in the coördinates of each point of $P_{6}^{2}$; that it vanishes at least once for each coincidence $\delta_{i j}$; that it vanishes twice for the coincidences $\delta_{12}, \delta_{13}, \delta_{23} ; \delta_{45}, \delta_{46}, \delta_{56}$; and that it vanishes with the factors $\delta, \delta_{123}$, and $\delta_{456}$ of $\Delta$. It corresponds therefore to the product of the 9 discriminant factors associated with the 9 double sixes in the complex $\Gamma_{123,456}$. In order to derive the remaining nine "complex invariants" of this type

* All of these configurations of lines are well known; cf. Pascal, loc. cit., II, pp. 284-90.

$\dagger$ If $P_{6}^{2}$ is taken in the canonical form (1) the same exception applies to $\delta_{234}, \delta_{134}, \delta_{124}$, and $\delta_{123}$. A closer study of the invariants of $G_{6,2}$ in $\Sigma_{4}$ would reveal the singular manifolds which correspond to these factors of $\Delta$ as well as to factors of type $\delta_{i} ;$. This information however is not necessary for our purposes. 
by permutation of the points* we shall complete the definition (16) by the further requirements:

$$
\begin{aligned}
\gamma_{i j k, l m n} & =\gamma_{j i k, l m n}=\gamma_{j k i, l m n} \\
& =\gamma_{i j k, m l n}=\gamma_{i j k, m n l}=-\gamma_{l m n, i j k} .
\end{aligned}
$$

Then the ten complex invariants of the first type are

TABLE I

$$
\begin{array}{ll}
\gamma_{123,456}=d_{2}(123)(456), & \gamma_{125,463}=d_{2}(125)(463), \\
\gamma_{134,562}=d_{2}(134)(562), & \gamma_{136,524}=d_{2}(136)(524), \\
\gamma_{145,623}=d_{2}(145)(623), & \gamma_{142,635}=d_{2}(142)(635), \\
\gamma_{156,234}=d_{2}(156)(234), & \gamma_{153,246}=d_{2}(153)(246), \\
\gamma_{162,345}=d_{2}(162)(345), & \gamma_{164,352}=d_{2}(164)(352) .
\end{array}
$$

From equations I (34) we get at once the values of the $\gamma_{i j k, l m n}$ in terms of $d_{2}, \bar{a}, \cdots, \bar{f}$.

Consider again the product

$$
\gamma_{i j, k l, m n}=(i k l)(j k l)(k m n)(l m n)(m i j)(n i j) .
$$

It also is of degree 3 in the coördinates of each point of $P_{6}^{2}$; it vanishes at least once for every coincidence; it vanishes twice for the coincidences $\delta_{i j}$, $\delta_{k l}$, and $\delta_{m n}$; it vanishes along with the six factors $\delta_{i k l}$, etc., of $\Delta$; and it therefore corresponds as above to the complex $\Gamma_{i j, k l, m n}$. Its definition is completed by the further requirements (which accord with permutations of the points of $\left.P_{6}^{2}\right)$ :

$$
\gamma_{i j, k l, m n}=\gamma_{k i, m n, i j}=\gamma_{j i, k l, m n} \neq \gamma_{i j, m n, k l} .
$$

Hence there are 30 complex invariants of this type which occur in 15 pairs.

In order to get explicit expressions for them in terms of $d_{2}, \bar{a}, \cdots, \bar{f}$ consider the particular pair $\gamma_{12,34,55}$ and $\gamma_{12,56,34}$. If in these we substitute respectively for $(134)(356)$ and (256)(412) their values (315) (364) $+(316)(345)$ and $(264)(215)+(245)(216)$ we find that

$$
\begin{aligned}
& \gamma_{12,34,56}=(364)(512) \cdot(234)(456)(612)(315) \\
& +(345)(612) \cdot(234)(456)(512)(316), \\
& \gamma_{12,56,34}=(364)(512) \cdot(156)(312)(534)(264) \\
& +(345)(612) \cdot(156)(132)(634)(245) .
\end{aligned}
$$

* We use here the parallel generating permutations of $I(28)$, viz.:

$$
\text { (12), (23456); ( } \bar{a} \bar{d})(\bar{b} \bar{e})(\bar{c} \bar{f}),(\bar{a} \bar{d} \bar{b} \bar{f} \bar{e}),
$$

an odd permutation requiring also a change of sign in $d_{2}$. 
From I (46) and (47) we have*

$$
\begin{aligned}
& (234)(456)(612)(315)+(156)(312)(534)(264)=\begin{array}{c}
\overline{d e} \\
-d_{2},
\end{array} \\
& (234)(456)(512)(316)+(156)(132)(634)(245)=\begin{array}{l}
-\overline{a c} \\
-d_{2}
\end{array} . \\
& \therefore \gamma_{12,34,56}-\gamma_{12,56,34}=-d_{2}[(364)(512)+(345)(612)] \\
& =d_{2}(\bar{d}-\bar{a}) \text {. } \\
& \gamma_{12,34,56}+\gamma_{12,56,34}=(\bar{a}+\bar{c}+\bar{e}) \overline{d e}+(\bar{d}+\bar{c}+\bar{e}) \overline{a c} \\
& =(\bar{a}+\bar{c}+\bar{e})\left(a_{2}+2 \bar{d}^{2}+2 \bar{e}^{2}+2 \bar{d} \bar{e}\right) \\
& +(\bar{d}+\bar{c}+\bar{e})\left(a_{2}+2 \bar{a}^{2}+2 \bar{c}^{2}+2 \bar{a} \bar{c}\right) \\
& =-a_{2}(\bar{a}+\bar{d})-2\left(\bar{a}^{3}+\bar{d}^{3}\right)+2\left[a_{2} \sigma_{1}+\Sigma \bar{a}^{3}+\Sigma \bar{a}^{2} \bar{d}+\sigma_{3}\right] \\
& =-a_{2}(\bar{a}+\bar{d})-2\left(\bar{a}^{3}+\bar{d}^{3}\right)+2\left[a_{2} \sigma_{1}+\sigma_{1}^{3}-2 \sigma_{1} \sigma_{2}+\sigma_{3}\right],
\end{aligned}
$$

where the $\sum$ 's refer to symmetric functions and the $\sigma$ 's to elementary symmetric functions of $\bar{a}, \bar{c}, \bar{d}, \bar{e}$. But from

$$
\begin{gathered}
\bar{b}+\bar{f}=-\sigma_{1}, \quad \bar{b} \bar{f}+(\bar{b}+\bar{f}) \sigma_{1}=a_{2}-\sigma_{2}, \\
\bar{b} \bar{f} \sigma_{1}+(\bar{b}+\bar{f}) \sigma_{2}=a_{3}-\sigma_{3}
\end{gathered}
$$

we get by eliminating $\bar{b}+\bar{f}$ and $\bar{b} \bar{f}$ the relation

$$
\begin{gathered}
a_{2} \sigma_{1}+\sigma_{1}^{3}-2 \sigma_{1} \sigma_{2}+\sigma_{3}-a_{3}=0 . \\
\therefore \gamma_{12,34,56}+\gamma_{12,56,34}=2 a_{3}-a_{2}(\bar{a}+\bar{d})-2\left(\bar{a}^{3}+\bar{d}^{3}\right) .
\end{gathered}
$$

If now we introduce the new set of six quantities $\bar{\alpha}, \cdots, \bar{\zeta}$ defined by

which satisfy the relation

$$
\bar{\alpha}=a_{3}-a_{2} \bar{a}-2 \bar{a}^{3}
$$

$$
\bar{\alpha}+\bar{\beta}+\bar{\gamma}+\bar{\delta}+\bar{\epsilon}+\bar{\zeta}=0,
$$

then

$$
\gamma_{12,34,56}+\gamma_{12,56,34}=\bar{\alpha}+\bar{\delta} . \dagger
$$

* The formulæ used here are derived from those quoted by proper permutation.

$\dagger$ These six values which serve so well for the expression of the complex invariants have another interesting contact. If $\bar{a}, \cdots, \bar{f}$ are the roots of a sextic then (21) is the Tschirnhausen transformation to a sextic with roots $\bar{\alpha}, \cdots, \bar{\zeta}$. If $d_{2}=0$, i. e., if $a_{2}^{2}-4 a_{4}=0$, the first sextic is the sextic of Maschke and the transformed sextic is that of Joubert. For it is easy to verify that $\Sigma \bar{\alpha}^{3}=9 .\left(a_{2}^{2}-4 a_{4}\right)\left(a_{2} a_{3}-2 a_{5}\right)=q_{4} q_{5}$. From this point of view the transformation (21) has been used in an earlier paper-Coble, An application of Moore's cross-ratio group, etc.; these T r a n s a c t i o n s, vol. 12 (1911), p. 323, (32); cited hereafter as $\mathbf{C 1}$. 
Combining this with (20) we get values of $\gamma_{12,34,56}$ and $\gamma_{12,56,34}$ and by permutation obtain the values of the 30 complex invariants of the second type. These are collected in

$$
\begin{gathered}
\text { TABLE II* } \\
2 \gamma_{12,34,56}=\bar{\alpha}+\bar{\delta}-d_{2}(\bar{a}-\bar{d}), \\
2 \gamma_{12,56,34}=\bar{\alpha}+\bar{\delta}+d_{2}(\bar{a}-\bar{d}),
\end{gathered}
$$

The 40 complex invariants are connected by many relations. We see at once that the 10 invariants in Table I are connected by 15 relations of the type:

$1^{\circ}$.

$$
\gamma_{123,456}+\gamma_{124,356}+\gamma_{134,256}+\gamma_{234,156}=0 .
$$

The complexes which correspond to the four terms are those which contain the syzygetic duad of double sixes $D, D_{56}$. Corresponding therefore to the four other types of syzygetic duads listed above we have respectively four other types of relations:

$2^{\circ}$.

$$
\begin{array}{ll}
2^{\circ} . & \gamma_{23,15,64}+\gamma_{12,35,64}-\gamma_{42,65,13}-\gamma_{62,45,13}=0, \\
3^{\circ} . & \gamma_{456,123}+\gamma_{45,61,23}+\gamma_{45,62,31}+\gamma_{45,63,12}=0, \\
4^{\circ} . & \gamma_{123,456}+\gamma_{23,14,56}+\gamma_{31,24,56}+\gamma_{12,34,56}=0, \\
5^{\circ} . & \gamma_{12,34,56}-\gamma_{12,56,34}+\gamma_{156,234}-\gamma_{134,256}=0 .
\end{array}
$$$$
4^{\circ} \text {. } \quad \gamma_{123,456}+\gamma_{23,14,56}+\gamma_{31,24,56}+\gamma_{12,34,56}=0 \text {, }
$$

These 270 linear relations can be verified by using the above five typical ones in connection with Tables I-II.

Not more than 10 of the 40 complex invariants are linearly independent, for they are expressed above in terms of $d_{2} \bar{a}, \cdots, d_{2} \bar{f}, \bar{\alpha}, \cdots, \bar{\zeta}$ where $d_{2} \sum \bar{a}=0$ and $\sum \bar{\alpha}=0$. That precisely 10 are independent can be verified by computing $d_{2}(\bar{a}-\bar{b}), \cdots, d_{2}(\bar{a}-\bar{f}), \bar{\alpha}-\bar{\beta}, \cdots, \bar{\alpha}-\bar{\zeta}$, and checking their independence.

The group $G_{6,2}$ is generated by transpositions of the points and by the element $A_{123}$. The effect of the transpositions upon the complex invariants can be inferred from the subscript notation in their definitions (16), (17) and (18), (19). The effect of the quadratic transformation $A_{123}$ upon the double sixes and the complexes is easily given. The element $A_{123}$ can at most permute the complex invariants to within outstanding factors. It must however transform the identities $1^{\circ}, \cdots, 5^{\circ}$ into identities. If then this outstanding factor is taken to be unity for some one invariant the value of the factor for other invariants can be inferred from the fact that identities involving this one invariant must be transformed into other identities. In

* The table can be completed so readily that it is not given here in full. However a complete table is necessary for checking some of the relations given below. 
this way the effect of the involution $A_{123}$ upon the double sixes, the complexes, and the complex invariants is found to be:

$$
\left(D_{123}\right),\left(D, D_{456}\right),\left(D_{124}, D_{34}\right),\left(D_{145}\right),\left(D_{12}\right),\left(D_{56}\right) \text {; }
$$

$(23)\left(\Gamma_{123,456}\right),\left(\Gamma_{124,356}, \Gamma_{12,34,56}\right),\left(\Gamma_{14,25,36}, \Gamma_{16,35,24}\right),\left(\Gamma_{12,56,34}\right)$;

$\left(\gamma_{123,456}\right),\left(\gamma_{124,356}, \gamma_{12,34,56}\right),\left(\gamma_{14,25,36},-\gamma_{16,35,24}\right),\left(\gamma_{12,56,34}\right)$.

Of course in these permutations (23) only typical cycles with reference to the division 123, 456 are given. The permutation of the $D$ 's is even while that of the $\Gamma$ 's is odd.

The complex invariants when expressed in terms of the coördinates $x, y$, $z, t, u$ of $P$ in $\Sigma_{4}$ lie in a linear system of dimension 9 of spreads invariant under $G_{6,2}$. Moreover they constitute the simplest linear system of this sort. For a member of such a system invariant under $G_{6 !}$ must be expressible in terms of $\bar{a}, \cdots, \bar{f}$. These are quadrics (I, p. 196) on the canonical basis of $\Sigma_{4}$. On the other hand a spread invariant under $A_{123}$ must be of order $5 k$ with $3 k$-fold points at the reference basis in $\Sigma_{4}$. The simplest way to reconcile these requirements is to take functions of the third degree in $\bar{a}, \cdots, \bar{f}$ from which $u$ factors out. Since $d_{2}$ contains the factor $u$ the invariants of Table I have this factor. The factor $u$ appears also in (156), (256), (125), (126) and every complex invariant of the type (18) must contain at least one of these factors. The word "simplest" as used above means of lowest degree in the coördinates of $P_{6}^{2}$.

(24) The 40 complex invariants satisfy a set of 270 four termed linear relations by means of which they reduce to 10 linearly independent invariants. The latter determine the simplest linear system of irrational invariants of $C^{(3)}$ and, as quintic spreads in $\Sigma_{4}$, the simplest linear system invariant under $G_{6,2}$. If $\Sigma_{4}$ be mapped upon an $S_{9}$ by means of this linear system $G_{6,2}$ appears in $S_{9}$ as a collineation group $\gamma_{51840}$.

In order to prove that the group $\gamma_{51840}$ in $S_{9}$ has an invariant pair of skew $\mathrm{S}_{4}$ 's we shall derive two new sets each containing 45 irrational "tritangent" invariants. A syzygetic n-ad of double sixes is a set of $n$ double sixes such that any two are syzygetic. The typical forms of the syzygetic tetrads are

$$
D, D_{i j}, D_{k l}, D_{m n} ; \quad D_{i j k}, D_{i l m}, D_{n j l}, D_{n k m} ; \quad D_{i j}, D_{k l}, D_{i j m}, D_{k l m} ;
$$

there being 15, 30, 90 respectively of each kind. The syzygetic duads and triads can all be picked from these tetrads. The 135 syzygetic tetrads divide into 45 sets $T$ of three such that, in any set of three, double sixes from different tetrads are azygetic. Any tetrad lies in but one set $T$ since the 8 double sixes in the other two tetrads of $T$ comprise all that are azygetic with the four 
members of the given tetrad. The typical forms of the sets $T$ are

$$
\begin{gathered}
T_{i j, k l, m n} \equiv D, D_{i j}, D_{k l}, D_{m n} ; D_{i k m}, D_{i l n}, D_{j k n}, D_{j l m} ; \\
D_{j k m}, D_{j l n}, D_{i k n}, D_{i k m} ; \\
T_{i j k l, m} \equiv D_{i j}, D_{k l}, D_{i j m}, D_{k l m} ; \quad D_{i k}, D_{j l}, D_{i k m}, D_{j l m} ; \\
D_{i l}, D_{j k}, D_{i l m}, D_{j k m} ;
\end{gathered}
$$

there being 15 of the first kind and 30 of the second kind. The 48 lines of $C^{(3)}$ in a syzygetic tetrad consist of 24 lines each occurring twice, so that three lines of $C^{(3)}$ in a tritangent plane are omitted from a tetrad and the same three lines are omitted from each of the tetrads in $T$, whence the sets $T$ correspond to the tritangent planes.

A syzygetic triad can be enlarged in but one way into a syzygetic tetrad, whence there are 135.4 syzygetic triads. The four complexes in each of the 270 relations $1^{\circ}, \cdots, 5^{\circ}$ each have a common syzygetic duad, whence all the relations are conjugate. We see from $1^{\circ}$ that any two complexes in such a relation have a common syzygetic triad, whence any two terms must occur in three relations. The two complementary pairs drawn from a relation determine two syzygetic triads drawn from the same tetrad, whence given a tetrad the four triads formed from it determine four pairs of terms and by equating these pairs of terms six relations are obtained. Thus the 135 tetrads determine 135.6 relations, but each is obtained three times, corresponding to its three pairs of pairs of terms.

Let us select the particular set $T_{12,34,56}$ and write down for each of its tetrads the four equal pairs of terms. We find with the help of Table I and II that

$$
\begin{aligned}
d_{2}(\bar{d}-\bar{a}) & =\gamma_{12,34,56}-\gamma_{12,56,34}=\gamma_{134,256}-\gamma_{156,234} \\
& =\gamma_{356,412}-\gamma_{312,456}=\gamma_{512,634}-\gamma_{534,612} \\
\frac{1}{2}\left[\bar{\alpha}-\bar{\delta}+d_{2}(\bar{a}-\bar{d})\right] & =\gamma_{13,46,25}-\gamma_{16,35,24}=\gamma_{14,35,26}-\gamma_{15,46,23} \\
& =\gamma_{15,24,36}-\gamma_{13,26,45}=\gamma_{16,23,45}-\gamma_{14,25,63} \\
\frac{1}{2}\left[\bar{\delta}-\bar{\alpha}+d_{2}(\bar{a}-\bar{d})\right] & =\gamma_{14,36,25}-\gamma_{16,45,23}=\gamma_{13,45,26}-\gamma_{15,36,24} \\
& =\gamma_{15,23,46}-\gamma_{14,26,35}=\gamma_{16,24,35}-\gamma_{13,25,64}
\end{aligned}
$$

The sum of these three "tetrad invariants" is zero and we form from them as follows the pair of "tritangent invariants":

$$
\begin{aligned}
2\left(\rho-\rho^{2}\right) t_{12,34,56} & =2\left[d_{2}(\bar{d}-\bar{a})\right]+\rho\left[\bar{\alpha}-\bar{\delta}+d_{2}(\bar{a}-\bar{d})\right] \\
& +\rho^{2}\left[\bar{\delta}-\bar{\alpha}+d_{2}(\bar{a}-\bar{d})\right], \\
(26) 2\left(\rho^{2}-\rho\right) \tau_{12,34,56}=2\left[d_{2}(\bar{d}-\bar{a})\right]+\rho^{2}\left[\bar{\alpha}-\bar{\delta}+d_{2}(\bar{a}-\bar{d})\right] & +\rho\left[\bar{\delta}-\bar{\alpha}+d_{2}(\bar{a}-\bar{d})\right], \\
\rho & =e^{2 \pi i / 3} .
\end{aligned}
$$


Both $t_{12,34,56}$ and $\tau_{12,34,56}$ contain the factor $\bar{d}-\bar{a}$ since

$$
\bar{\alpha}-\bar{\delta}=(\bar{d}-\bar{a}) \overline{a d},
$$

and both vanish when the three lines of their corresponding tritangent plane of $C^{(3)}$ meet in a point.

In order to obtain the second type of tritangent invariant we carry out on the above type the transformation $A_{123}$ and interchange $t, \tau$ and $\rho, \rho^{2}$. Then $T_{12,34,56}$ becomes $T_{1256,4}$ and the three tetrad invariants associated with $T_{1256,4}$ become

$\gamma_{124,356}-\gamma_{12,56,34}, \quad \gamma_{16,23,45}+\gamma_{16,35,24}, \quad-\gamma_{15,34,26}-\gamma_{135,246}$.

These have the values

$$
\begin{aligned}
& -\frac{1}{2}\left[\bar{\alpha}+\bar{\delta}+d_{2}(\bar{b}+\bar{c}-\bar{e}-\bar{f})\right], \\
& -\frac{1}{2}\left[\bar{\beta}+\bar{\gamma}+d_{2}(\bar{e}+\bar{f}-\bar{a}-\bar{d})\right], \\
& -\frac{1}{2}\left[\bar{\epsilon}+\bar{\zeta}+d_{2}(\bar{a}+\bar{d}-\bar{b}-\bar{c})\right] .
\end{aligned}
$$

As we should expect their sum is zero. With multipliers $1, \rho, \rho^{2}$ respectively we form $\left(\rho^{2}-\rho\right) \tau_{1256,4}$; and with multipliers $1, \rho^{2}, \rho$ we form $\left(\rho-\rho^{2}\right) t_{1256,4}$. Then

$$
\begin{aligned}
2 t_{1256,4}=\left[-\rho(\beta+\bar{\gamma})+\rho^{2}(\bar{\epsilon}+\bar{\zeta})\right. & -d_{2}(\bar{a}+\bar{d}) \\
& \left.-\rho^{2} d_{2}(\bar{b}+\bar{c})-\rho d_{2}(\bar{e}+\bar{f})\right], \\
2 \tau_{1256,4}=\left[-\rho^{2}(\bar{\beta}+\bar{\gamma})+\rho(\bar{\epsilon}+\bar{\zeta})\right. & -d_{2}(\bar{a}+\bar{d}) \\
& \left.-\rho d_{2}(\bar{b}+\bar{c})-\rho^{2} d_{2}(\bar{e}+\bar{f})\right] .
\end{aligned}
$$

We shall now make an important change of variables. Let

$$
\begin{array}{lll}
x_{a}=\bar{\alpha}+\left(\rho-\rho^{2}\right) d_{2} \bar{a}, & \cdots, & x_{f}=\bar{\zeta}+\left(\rho-\rho^{2}\right) d_{2} \bar{f}, \\
u_{a}=\bar{\alpha}+\left(\rho^{2}-\rho\right) d_{2} \bar{a}, & \cdots, & u_{f}=\bar{\zeta}+\left(\rho^{2}-\rho\right) d_{2} \bar{f} .
\end{array}
$$

Then the two types of tritangent invariants and the three types of complex invariants take the following very simple forms:

$$
\begin{gathered}
2 t_{12,34,56}=x_{a}-x_{d}, \\
2 \tau_{12,34,56}=u_{a}-u_{d} . \\
2 t_{1256,4}=-\rho\left(x_{b}+x_{c}\right)+\rho^{2}\left(x_{e}+x_{f}\right), \\
2 \tau_{1256,4}=-\rho^{2}\left(u_{b}+u_{c}\right)+\rho\left(u_{e}+u_{f}\right) . \\
2\left(\rho-\rho^{2}\right) \gamma_{123,456}=x_{a}+x_{b}+x_{c}-u_{a}-u_{b}-u_{c}, \\
2\left(\rho-\rho^{2}\right) \gamma_{12,34,56}=\rho x_{a}-\rho^{2} x_{d}-\rho^{2} u_{a}+\rho u_{d}, \\
2\left(\rho-\rho^{2}\right) \gamma_{12,56,34}=-\rho^{2} x_{a}+\rho x_{d}+\rho u_{a}-\rho^{2} u_{d} .
\end{gathered}
$$


The new variables are subject to the relations

$$
x_{a}+\cdots+x_{f}=0, \quad u_{a}+\cdots+u_{f}=0 .
$$

Their permutations under the operations of $G_{6 !}$ are evident at once from (30). In order to determine the effect upon them of the element $A_{123}$ we need only to take the transformation (23), translate it to the new variables by using (33), and express the transformed variables in terms of the original ones. This presents no difficulty and the result is

$$
\begin{aligned}
& 6 x_{a}^{\prime}=\left(-3 \rho^{2}+\rho\right) u_{a}+\left(3 \rho^{2}+\rho\right)\left(u_{b}+u_{c}\right)+\left(u_{d}+u_{e}+u_{f}\right), \\
& 6 x_{b}^{\prime}=\left(-3 \rho^{2}+\rho\right) u_{b}+\left(3 \rho^{2}+\rho\right)\left(u_{c}+u_{a}\right)+\left(u_{d}+u_{e}+u_{f}\right), \\
& 6 x_{c}^{\prime}=\left(-3 \rho^{2}+\rho\right) u_{c}+\left(3 \rho^{2}+\rho\right)\left(u_{a}+u_{b}\right)+\left(u_{d}+u_{e}+u_{f}\right), \\
& 6 x_{d}^{\prime}=\left(u_{a}+u_{b}+u_{c}\right)+\left(-3 \rho+\rho^{2}\right) u_{d}+\left(3 \rho+\rho^{2}\right)\left(u_{e}+u_{f}\right), \\
& 6 x_{e}^{\prime}=\left(u_{a}+u_{b}+u_{c}\right)+\left(-3 \rho+\rho^{2}\right) u_{e}+\left(3 \rho+\rho^{2}\right)\left(u_{f}+u_{d}\right), \\
& 6 x_{f}^{\prime}=\left(u_{a}+u_{b}+u_{c}\right)+\left(-3 \rho+\rho^{2}\right) u_{f}+\left(3 \rho+\rho^{2}\right)\left(u_{d}+u_{e}\right) .
\end{aligned}
$$

The transformation is completed by assigning to the matrix of the transformation which expresses $6 u_{a}^{\prime}, \cdots, 6 u_{f}^{\prime}$ in terms of $x_{a}, \cdots, x_{f}$ coefficients which are conjugate to those of the matrix in (35).

The most important result which appears from this form of $A_{123}$ is that the variables $x$ and $u$ are interchanged. It is clear from (30) that the same is true of the transpositions of $G_{6 !}$. Hence the space $S_{4}$ defined in $S_{9}$ by

$$
u_{a}=\cdots=u_{f}=0 \text {, }
$$

in which $x_{a}, \cdots, x_{f}$ are variables and the space $\bar{S}_{4}$ defined in $S_{9}$ by

$$
x_{a}=\cdots=x_{f}=0,
$$

in which $u_{a}, \cdots, u_{f}$ are variables, are either unaltered or interchanged by an operation of $\gamma_{51840}$ according as this operation can be expressed as a product of an even or an odd number of the 36 generating involutions. Hence

(36) The collineation group $\gamma_{51840}$ of (24) has an invariant pair of skew $S_{4}$ 's. Its invariant subgroup $\gamma_{25920}$ appears in either $S_{4}$ as a collineation group with a conjugate set of 40 linear spaces (33) and a conjugate set of 45 linear spaces ((32), (31)).

It is clear from (17), (19), and (23) that

$$
\sum_{10} \gamma_{123,456}^{2} *
$$

is an invariant of $G_{6,2}$ if it does not vanish identically. From (33) we have $-12 \sum_{40} \gamma_{123,456}^{2}=5 \sum_{6} x_{a}^{2}+5 \sum_{6} u_{a}^{2}+4 \sum_{15} x_{a} x_{b}+4 \sum_{15} u_{a} u_{b}+6 \sum_{30} x_{a} u_{b}$.

\footnotetext{
* The number under a summation sign is the number of terms used in the sum.
} 
If we make use of (34) we find that

$$
\sum_{40} \gamma_{123,456}^{2}=2 \sum_{6} x_{a} u_{a} .
$$

The expression for this invariant in terms of $\bar{a}, \cdots, \bar{f}$ is from (30):

$$
\sum_{6} x_{a} u_{a}=\sum_{b}\left(\bar{\alpha}^{2}+3 d_{2}^{2} \bar{a}^{2}\right) .
$$

If we square $\bar{\alpha}$ and use a table of symmetric functions we find in the notation of $(9), \S 1$, that

$$
\begin{gathered}
\sum_{6} \bar{\alpha}^{2}=2 I_{6}+6 a_{2} q_{4} . \\
\therefore \sum_{40} \gamma_{123,456}^{2}=2 \sum_{6} x_{a} u_{a}=4 I_{6} .
\end{gathered}
$$

From this result there follows that under $\gamma_{25920}$ the variables $x$ and $u$ are contragredient. If then $x$ is a point in $S_{4}, u$ may be regarded as an $S_{3}$ in $S_{4}$ and the $x, u$ we shall define to be a counter-point in $S_{4}$. Then the elements of $\gamma_{51840}$ not in $\gamma_{25920}$ are correlations in $S_{4}$ since they interchange the members of counter-points and have the invariant (38). Thus $\gamma_{51840}$ can be regarded as a correlation group in $S_{4}, \gamma_{25920}$ as the invariant collineation subgroup of this correlation group.

This collineation group in $S_{4}$ is reasonably well identified with the Burkhardt group in $S_{4}$ by the two conjugate sets described in (36). To do this more precisely we observe with Burkhardt (BII, § 45) that his group can be so represented that it contains a subgroup $G_{6}$ ! which permutes symmetrically $6 S_{3}$ 's.* If this transformation be applied to his simplest invariant $J_{4}$ (BII, p. 208) it takes the form

$$
J_{4}=\sum_{15} x_{a} x_{b} x_{c} x_{d} \quad\left(x_{a}+\cdots+x_{f}=0\right) .
$$

I had noted in using another canonical form $\dagger$ that this quartic spread has a conjugate set of 45 double points. The spread (39) has double points of types

$$
1,-1,0,0,0,0 ; 1,1, \rho, \rho, \rho^{2} \rho^{2} .
$$

If we compare these coördinates with the coefficients of the tritangent invariants in (31) and (32), after roodifying $2 \tau_{1256,4}$ by adding

$$
-\frac{1}{3}\left(\rho-\rho^{2}\right)\left(u_{u}+\cdots+u_{f}\right)
$$

so that the sum of its coefficients is zero, we find them to be the same. Hence the group of the 45 double points is the group of the 45 tritangent invariants $\tau$.

(40) The Cremona group $G_{6,2}$ in, $\Sigma_{4}$ effects within its simplest invariant linear system a group of linear transformations $\gamma_{51840}$ which permutes the irrational

* Such a subgroup (one of 36 ) is generated by the elements $(12) \cdot I, \cdots,(56) \cdot I$ of $\Gamma_{6,2}$.

$\dagger$ Coble, An invariant condition for certain automorphic algebraic forms, A m e ri c a n Journal of Mathematics, vol. 28 (1906). 
invariants of $C^{(3)}$ in this linear system as the counter-points in $S_{4}$ are permuted under the operations of the* correlation group of order 51840 built upon Burkhardt's collineation group $G_{25920}$.

The group $\gamma_{51840}$ in $S_{9}$ has the invariant (38) which may be regarded as a quadric and which determines in $S_{9}$ a polarity $P$. This polarity and $\gamma_{51840}$ generate a correlation group $\gamma_{2.51840}$ in $S_{9}$ which has for invariant subgroups both $\gamma_{51840}$ and the $G_{2}$ whose elements are $1, P$. Any element of $\gamma_{51840}$ is permutable with $P$ whence any involution of $\gamma_{51840}$ multiplied by $P$ is a polarity $Q$. A convenient way to indicate any involution of $\gamma_{51840}$ is therefore to write the equation of the quadric $Q$. The involution is then $Q P$, a product which can be written at once because of the simple form of $P$.

In particular the conjugate set of 36 generating involutions of $\gamma_{51840}$ give rise to the set of $Q$ 's:

$$
\begin{aligned}
\frac{1}{6} Q= & x_{a}^{2}+\cdots+x_{f}^{2}+u_{a}^{2}+\cdots+u_{f}^{2}, \\
\frac{1}{6} Q_{12}= & -2\left(x_{a} x_{d}+x_{b} x_{e}+x_{c} x_{f}\right)-2\left(u_{a} u_{d}+u_{b} u_{e}+u_{c} u_{f}\right), \\
\frac{1}{6} Q_{456}= & -2 \rho^{2}\left(x_{b} x_{c}+x_{c} x_{a}+x_{a} x_{b}\right)-2 \rho\left(x_{e} x_{f}+x_{f} x_{d}+x_{d} x_{e}\right) \\
& -2 \rho\left(u_{b} u_{c}+u_{c} u_{a}+u_{a} u_{b}\right)-2 \rho^{2}\left(u_{e} u_{f}+u_{f} u_{d}+u_{d} u_{e}\right) .
\end{aligned}
$$

It is to be observed that the 36 products $P Q$, which in $S_{9}$ are collineations, in $S_{4}$ are correlations, and in fact polarities. The quadrics in $S_{4}$ which determine these 36 polarities in $S_{4}$ which generate the correlation group (40) are precisely those of (41) where however each $Q$ is the sum of the point-equation and the $S_{3}$-equation of the same quadric.

The following theorem, naturally of prime importance, is a consequence of (40), (30), and $\S 1$ (13).

(42) Any invariant of the Burkhardt group $G_{25920}$ of total degree $2 k$ in $x$ and $u$, or the sum or difference of two dual invariants of such total degree, is an invariant of $C^{(3)}$ of degree $6 k$ in $\bar{a}, \cdots, \bar{f}$, which is rational in $I_{6}, I_{12}, \sqrt{\Delta}, I_{18}$, and $I_{30}$.

In this way invariants of the hexahedral surface can be calculated. For example, we have already found in (38) that the invariant $\sum_{6} x_{a} u_{a}$ of $G_{29520}$ gives rise to the invariant $2 I_{6}$ of $C^{(3)}$. We find also that the invariant $J_{4}$ of (39) and its dual give rise to the following invariants of $C^{(3)}$ :

$$
\begin{gathered}
6\left[\sum_{15} x_{a} x_{b} x_{c} x_{d}+\sum_{15} u_{a} u_{b} u_{c} u_{d}\right]=4 I_{6}^{2}+24 a_{2} q_{4} I_{6} \\
+24 a_{2} q_{5}^{2}-288 a_{3} q_{4} q_{5}+80 a_{2}^{2} q_{4}^{2}-48 q_{4}^{3}, \\
6\left[\sum_{15} x_{a} x_{b} x_{c} x_{d}-\sum_{15} u_{a} u_{b} u_{c} u_{d}\right]=-40\left(\rho-\rho^{2}\right) \sqrt{\Delta} .
\end{gathered}
$$

The first of these might be taken as $I_{12}$, but it is to be remarked that invariants of $C^{(3)}$ obtained in this way are not in immediate relation with a system obtained by known covariant processes such as is contemplated in $\S 2$.

* Since $G_{25920}$ is simple it can be enlarged to a correlation group in only one way. Trans. Am. Math. Soc. 23 
4. The solution of the Form problem of $G_{6,2}$ IN terms of a solution OF BURKHARDT'S FORM PROBLEM

The data necessary for this purpose are a set of independent invariants of the Burkhardt group (which we shall take as given in BII with variables $\left.y_{0}, y_{1}, \cdots, y_{4}\right)$ and a set of five invariant mixed forms linear in the space coördinates $v_{0}, v_{1}, \cdots, v_{4}$. The expressions in $y$ for the invariants $J_{4}, J_{6}$, $J_{10}, J_{12}$, and $J_{18}$ given by Burkhardt seem open to suspicion (cf. footnotes, $\S \S 4,6)$ and we shall merely assume that independent invariants of the degrees indicated exist which together with their jacobian, $J_{45}$, constitute a complete system in the variables $y$ alone.

It is noted in BII, p. 218, that the forty squares

$$
\begin{array}{r}
9 y_{0}^{2}, \quad-3\left(y_{0}+2 \epsilon^{\lambda} y_{a}\right)^{2}, \quad\left(\epsilon=e^{2 \pi i / 3}\right),(\alpha=1, \cdots, 4), \\
\left(y_{0}+2 \epsilon^{\lambda} y_{1}+2 \epsilon^{\mu} y_{2}+2 \epsilon^{\nu} y_{3}+2 \epsilon^{-\lambda-\mu-\nu} y_{4}\right)^{2} \quad(\lambda, \mu, \nu=0,1,2),
\end{array}
$$

are merely permuted under the operations of the group. If then $J_{2 k}$ is the sum of the $k$ th powers of these squares, $J_{2 k}$ either vanishes or is an invariant. In order to prove that $J_{2 k}(k=2,3,5,6,9)$ together with $J_{45}$ constitute a complete system we have only to prove that their jacobian is not zero and therefore must be a numerical multiple of $J_{45}$. To simplify the calculation we shall assume after taking the derivatives that

$$
y_{0}=y_{4}=0, \quad y_{1}=-y_{2}=1, \quad y_{3}=t .
$$

Then $J_{45}$, the product of the 45 linear forms given in BII, p. 195, takes the simple form

$$
J_{45}=\sigma t^{12}\left(t^{6}-1\right)\left(t^{6}+3^{3}\right)^{3},
$$

where $\sigma$ is a numerical constant. The first derivatives of $J_{2 k}$ for the given values of $k$ become

$$
\begin{aligned}
\frac{1}{2^{2 k-1}} \frac{1}{2 k} \frac{\partial J_{2 k}}{\partial y_{0}} & =\sum_{\lambda}(-3)^{k} \epsilon^{\lambda(2 k-1)} t^{2 k-1}+\sum_{\lambda, \mu, \nu}\left(\epsilon^{\lambda}-\epsilon^{\mu}+\epsilon^{\nu} t\right)^{2 k-1}, \\
\frac{1}{2^{2 k}} \frac{1}{2 k} \frac{\partial J_{2 k}}{\partial y_{1}} & =\sum_{\lambda}(-3)^{k} \epsilon^{2 k \lambda}+\sum_{\lambda, \mu, \nu}\left(\epsilon^{\lambda}-\epsilon^{\mu}+\epsilon^{\nu} t\right)^{2 k-1} \epsilon^{\lambda}, \\
\frac{1}{2^{2 k}} \frac{1}{2 k} \frac{\partial J_{2 k}}{\partial y_{2}} & =\sum_{\lambda}-(-3)^{k} \epsilon^{2 k \lambda}+\sum_{\lambda, \mu, \nu}\left(\epsilon^{\lambda}-\epsilon^{\mu}+\epsilon^{\nu} t\right)^{2 k-1} \epsilon^{\mu}, \\
\frac{1}{2^{2 k}} \frac{1}{2 k} \frac{\partial J_{2 k}}{\partial y_{3}} & =\sum_{\lambda}(-3)^{k} \epsilon^{2 k \lambda} t^{2 k-1}+\sum_{\lambda, \mu, \nu}\left(\epsilon^{\lambda}-\epsilon^{\mu}+\epsilon^{\nu} t\right)^{2 k-1} \epsilon^{\nu}, \\
\frac{1}{2^{2 k}} \frac{1}{2 k} \frac{\partial J_{2 k}}{\partial y_{4}} & =\sum_{\lambda, \mu, \nu}\left(\epsilon^{\lambda}-\epsilon^{\mu}+\epsilon^{\nu} t\right)^{2 k-1} \epsilon^{-\lambda-\mu-\nu} .
\end{aligned}
$$

We see that for $k=2$ and $k=5$ only the first and last derivatives do not 
vanish so that the determinant of these derivatives is a factor of the jacobian which turns out to be

$$
-2^{3} \cdot 3^{7} \cdot 5^{2} t^{4}\left(t^{6}+3^{3}\right),
$$

which is a factor of $J_{45}$. The remaining factor is the determinant of the other derivatives for $k=3,6,9$. If we write these derivatives in order for a given $k$ in a column, then the first and second rows are interchanged with a change of sign throughout, if $\lambda$ and $\mu$ be interchanged and $t$ changed in sign. If then we take the sum and difference of the first two rows for new first two rows, $t^{3}$ factors from the new first row and $t^{5}$ factors from the last row. The factor $t^{12}$ of $J_{45}$ is now accounted for and the columns are of degrees $0,1,2$ in $t^{6}$. It will now be sufficient to prove that the coefficient of the highest power of $t^{6}$ is not zero. This is

$$
27^{3} \cdot 2^{2}\left|\begin{array}{ccc}
10 & \left(\begin{array}{c}
11 \\
2
\end{array}\right) & \left(\begin{array}{c}
17 \\
2
\end{array}\right) \\
-12 & -9\left(\begin{array}{c}
11 \\
5
\end{array}\right) & -9\left(\begin{array}{c}
17 \\
5
\end{array}\right) \\
1-3 & 1+3^{4} & 1-3^{7}
\end{array}\right|=2^{5} \cdot 3^{12} \cdot 5 \cdot 31 \cdot 4181 .
$$

(44) The invariants $J_{2 \cdot 2}, J_{2 \cdot 3}, J_{2 \cdot 5}, J_{2 \cdot 6}$, and $J_{2 \cdot 9}$ defined above, together with $J_{45}$, constitute a complete system for the Burkhardt group.

We shall indicate by $\boldsymbol{J}_{i, k}$ an invariant form of this group of degree $i$ in $y$ and of degree $k$ in the dual coördinates $v$. Then the group has an invariant $J_{0,4}$, the dual of $J_{4,0}$ found above. I had noted (see loc. cit., §3) that these two forms had the symmetrical property that the polar quadric of a given quadric as to either would have for polar as to the other the given quadric. Thus either form defines the other and if we take with Burkhardt

$$
J_{4,0}=y_{0}^{4}+8 y_{0}\left(y_{1}^{3}+\cdots+y_{4}^{3}\right)+48 y_{1} y_{2} y_{3} y_{4},
$$

then $J_{0,4}$ must be

$$
J_{0,4}=v_{0}^{4}+v_{0}\left(v_{1}^{3}+\cdots+v_{4}^{3}\right)+3 v_{1} v_{2} v_{3} v_{4} .
$$

If we operate with the polar cubic of $v$ as to $J_{0,4}$ on any invariant $J_{r, 0}$ the result, if not zero, is an invariant $J_{r-3,1}$. We shall now prove that

(45) If we operate with the polar cubic of $v$ as to $J_{0,4}$ upon the sums of powers $J_{2 k}$ for $k=2,5,6,8,9$ we obtain five forms $J_{1,1}, J_{7,1}, J_{9,1}, J_{13,1}$, and $J_{15,1}$ which in $v$ are linearly independent and have for determinant $J_{45}$.

The proof will be carried out as above. The coefficients of $v_{0}, \cdots, v_{4}$ in $J_{2 k-3,1}$ become after using the above special values of $y$ and taking out the numerical factor $2 k(2 k-1)(2 k-2) 2^{2 k-1} \cdot 3$

$$
\begin{array}{ll}
\sum_{\lambda}(-3)^{k} \epsilon^{2 k \lambda} t^{2 k-3} & +3 \sum_{\lambda, \mu, \nu}\left(\epsilon^{\lambda}-\epsilon^{\mu}+\epsilon^{\nu} t\right)^{2 k-3}, \\
\sum_{\lambda}(-3)^{k} \epsilon^{(2 k-1) \lambda} & +3 \sum_{\lambda, \mu, \nu}\left(\epsilon^{\lambda}-\epsilon^{\mu}+\epsilon^{\nu} t\right)^{2 k-3} \epsilon^{2 \lambda},
\end{array}
$$




$$
\begin{aligned}
\sum_{\lambda}-(-3)^{k} \epsilon^{(2 k-1) \lambda} & +3 \sum_{\lambda, \mu, \nu}\left(\epsilon^{\lambda}-\epsilon^{\mu}+\epsilon^{\nu} t\right)^{2 k-3} \epsilon^{2 \mu}, \\
\sum_{\lambda}(-3)^{k} \epsilon^{(2 k-1) \lambda} t^{2 k-3} & +3 \sum_{\lambda, \mu, \nu}\left(\epsilon^{\lambda}-\epsilon^{\mu}+\epsilon^{\nu} t\right)^{2 k-3} \epsilon^{2 \nu} \\
& +3 \sum_{\lambda, \mu, \nu}\left(\epsilon^{\lambda}-\epsilon^{\mu}+\epsilon^{\nu} t\right)^{2 k-3} \epsilon^{\lambda+\mu+\nu} .
\end{aligned}
$$

Again for $k=6,9$ the coefficients of $v_{1}, v_{2}, v_{3}$ vanish so that the determinant of the five forms contains as a factor the two-rowed determinant of coefficients of $v_{0}, v_{4}$ for $k=6,9$. This is easily reduced to

$$
\begin{aligned}
& 3^{6} \mid \begin{array}{l}
\left(3^{4}+3\right) t^{9}-2 \cdot 3^{3}\left(\begin{array}{c}
9 \\
3
\end{array}\right) t^{3} \\
\left(-3^{7}+3\right) t^{15}-2 \cdot 3^{3}\left(\begin{array}{l}
15 \\
9
\end{array}\right) t^{9}+2 \cdot 3^{6}\left(\begin{array}{c}
15 \\
3
\end{array}\right) t^{3}
\end{array} \\
& \begin{array}{l}
6\left(\begin{array}{c}
9 \\
4
\end{array}\right) t^{5}\left(\epsilon-\epsilon^{2}\right)^{4} \\
6\left(\begin{array}{c}
15 \\
4
\end{array}\right) t^{11}\left(\epsilon-\epsilon^{2}\right)^{4}+6\left(\begin{array}{c}
15 \\
10
\end{array}\right) t^{5}\left(\epsilon-\epsilon^{2}\right)^{10}
\end{array} \mid \\
& =3^{11} \cdot 2^{2} \cdot 7^{2} \cdot 13 t^{8}\left|\begin{array}{ll}
t^{6}-2 \cdot 3^{3} & 3 \\
-4 t^{12}-3^{2} \cdot 5 \cdot 11 t^{6}+3^{5} \cdot 5 & 5 t^{6}-3^{3} \cdot 11
\end{array}\right| \\
& =3^{11} \cdot 2^{2} \cdot 7^{2} \cdot 13 \cdot 17 t^{8}\left(t^{6}+3^{3}\right)^{2} .
\end{aligned}
$$

This is a factor of $J_{45}{ }^{*}$ There remains the determinant of the coefficients of $v_{1}, v_{2}, v_{3}$ for $k=2,5,8$ which reads as follows when we denote

$$
\begin{gathered}
\sum_{\lambda, \mu}\left(\epsilon^{\lambda}-\epsilon^{\mu}+t\right)^{k} \quad \text { by } s_{k}: \\
\left|\begin{array}{lll}
3^{3}+9 s_{1} \epsilon^{2 \lambda} & -3^{6}+9 s_{7} \epsilon^{2 \lambda} & 3^{9}+9 s_{13} \epsilon^{2 \lambda} \\
-3^{3}+9 s_{1} \epsilon^{2 \mu} & 3^{6}+9 s_{7} \epsilon^{2 \mu} & -3^{9}+9 s_{13} \epsilon^{2 \mu} \\
3^{3} t+9 s_{1} & -3^{6} t^{7}+9 s_{7} & 3^{9} t^{13}+9 s_{13}
\end{array}\right| .
\end{gathered}
$$

By adding and subtracting the first and second rows to form new rows we get the determinant

$$
\begin{aligned}
& 2^{4} \cdot 3^{11} t^{4}\left|\begin{array}{lll}
0 & -35 & -\left(\begin{array}{c}
13 \\
4
\end{array}\right) t^{6}+\left(\begin{array}{c}
13 \\
10
\end{array}\right) 3^{3} \\
1 & 3\left(7 t^{6}-36\right) & 3\left[\left(\begin{array}{l}
13 \\
1
\end{array}\right) t^{12}-\left(\begin{array}{c}
13 \\
7
\end{array}\right) t^{6}+4 \cdot 3^{5}\right] \\
1 & -3\left(8 t^{6}+18 \cdot 7\right) & 3 \cdot 244 t^{12}-2\left(\begin{array}{c}
13 \\
6
\end{array}\right) t^{6}+2 \cdot 13 \cdot 3^{6}
\end{array}\right| \\
& =2^{5} \cdot 3^{14} \cdot 5 \cdot 11 \cdot 19 t^{4}\left(t^{6}-1\right)\left(t^{6}+3^{3}\right)
\end{aligned}
$$

which is the remaining factor of $J_{45}$ and theorem (45) is established.

According to $\S 3(42)$ the invariants $J_{2 \cdot 2}, \cdots, J_{2 \cdot 9}$ of (44) give rise to in-

${ }^{*}$ I originally tried to derive a $J_{9,1}$ and $J_{15,1}$ by operating with the above polar cubic on Burkhardt's $J_{12}$ and $J_{18}$ as explicitly given in terms of the $y$ 's in BII, pp. 208-9. As above only the terms in $v_{0}, v_{4}$ persisted for $y_{0}=y_{4}=0$. But their two-rowed determinant could not be expressed as a factor of $J_{45}$. Either my own calculation was wrong or there is some mistake in one of these forms other than the misprint (p. 209) in the term $-8 \Sigma y_{1}^{6} y_{2}^{3} y_{3}^{3} y_{4}^{3}$ which should read $-8 \Sigma y_{1}^{9} y_{2}^{3} y_{3}^{3} y_{4}^{3}$. This was one of the reasons for introducing a new complete system. 
variants $I_{12}^{\prime}, I_{18}^{\prime}, I_{30}^{\prime}, I_{36}^{\prime}$, and $I_{54}^{\prime}$ of the group $\Gamma_{6,2}$ and the invariants $J_{1,1}$, $\cdots, J_{15,1}$ of (45) give rise to invariants $I_{6}^{\prime \prime}, I_{24}^{\prime \prime}, I_{30}^{\prime \prime}, I_{42}^{\prime \prime}$, and $I_{48}^{\prime \prime}$ of $\Gamma_{6,2}$. Explicit expressions for the invariants $I^{\prime}$ and $I^{\prime \prime}$ could be obtained if a complete system for $G_{6,2}$ had been selected. Their expressions in terms of $\bar{a}, \cdots, \bar{f}$ could easily be written, since they are given in terms of the sums of powers of the 40 linear forms in $x$ found in $\S 3$ (33) and from $\S 3$ (30), (21) the values of $x$ in terms of $\bar{a}, \cdots, \bar{f}$ are known.

The requisite steps in the solution of the form problem of $\Gamma_{6,2}$ when a solution of Burkhardt's form problem is adjoined are as follows:

$1^{\circ}$. From the given values of $I_{6}, I_{12}, \sqrt{\Delta}, I_{18}, I_{30}$ the values of $I_{12}^{\prime}, I_{18}^{\prime}$, $I_{30}^{\prime}, I_{36}^{\prime}, I_{54}^{\prime}$ above are determined and thereby the given values of the Burkhardt form problem are ascertained.

$2^{\circ}$. From these values a solution of the Burkhardt form problem is calculated.

This solution we suppose taken in the canonical form $x_{a}, \cdots, x_{f}$ where the factor of proportionality $\lambda$ in the $x$ 's is determined from $J_{2 \cdot 3} / J_{2 \cdot 2}$ to within sign.

$3^{\circ}$. The values of $I_{6}^{\prime \prime}, \cdots, I_{48}^{\prime \prime}$ are determined in terms of the invariants of $\Gamma_{6,2}$; and from the system of five linear equations

$$
J_{1,1}=I_{6}^{\prime \prime}, \quad \cdots, \quad J_{15,1}=I_{48}^{\prime \prime}
$$

together with $u_{a}+\cdots+u_{f}=0$, the values of $u_{a}, \cdots, u_{f}$ are found in terms of the $I^{\prime \prime}$ and of $x_{a}, \cdots, x_{f}$ to within the same factor $\lambda$.

$4^{\circ}$. From the equations

$$
x_{a}-u_{a}=2\left(\rho-\rho^{2}\right) d_{2} \bar{a}, \quad \cdots, \quad x_{f}-u_{f}=2\left(\rho-\rho^{2}\right) d_{2} \bar{f},
$$

values of $\mu \bar{a}, \cdots, \mu \bar{f}$ are obtained. If these values are used in place of $\bar{a}$, $\cdots, \bar{f}$ to determine $\bar{\alpha}$ in $x_{a}+u_{a}=2 \bar{\alpha}$, the value of $\mu^{3}$ is rationally determined. If in (8) $\S 1$ we use $\mu \bar{a}$, etc., rather than $\bar{a}$, etc., in forming $\Pi$ we have

$$
\sqrt{\Delta}=\frac{d_{2} \Pi_{\mu}}{\mu^{10}}=\frac{d_{2} \mu^{2} \Pi_{\mu}}{\mu^{12}}
$$

whence $d_{2} \mu^{2}$ is rationally determined.

$5^{\circ}$. The ratios of the coördinates of the point $P$ in $\Sigma_{4}$ which is a solution of $\Gamma_{6,2}$ are obtained from I, $\S 10$ (89) by replacing $\bar{a}, \cdots, \bar{f}, \rho$ by $\mu \bar{a}, \cdots, \mu \bar{f}$, $\mu \rho$ where $\mu \rho$ is rational in $\mu \bar{a}, \cdots, \mu \bar{f}, \mu^{2} d_{2}$.

The above procedure is valid except in the two cases when either $\Delta$ vanishes, or $J_{45}$, the determinant of the system of linear equations in $3^{\circ}$, vanishes. If $\Delta$ vanishes the lines of $C^{(3)}$ can be determined by the solution of a general sextic equation and the above apparatus is unnecessary. If $J_{45}$ vanishes then one of the tritangent invariants $t$ of $\S 3(26)$, (29) must vanish. The product of these is an invariant $I_{75} \cdot I_{60}$ of $\Gamma_{6,2}$. Without stopping to discuss 
the geometric meaning of $I_{60}$ it is clear that the vanishing of $J_{45}$ must imply the isolation of a tritangent plane of $C^{(3)}$ in which case as noted before the equations of the lines can be expressed by means of radicals.

That the Burkhardt form problem has precisely 25920 solutions shows that the form problem of $\Gamma_{6,2}$ has 25920 solutions and therefore that of $G_{6,2}$ has 51840 solutions. For any solution for $\Gamma_{6,2}$ leads to a solution for $G_{25920}$ and conversely. We now have to consider further only those processes involved in $2^{\circ}$.

\section{The NORMal hyperelliptic SURFace in $S_{8}$ OF Genus two AND GRADE THREE}

In this paragraph we shall develop certain geometrical facts concerning the hyperelliptic surface and its projections which suggest a solution of the Burkhardt form problem for the special case $J_{4}=0$.

It is known* that there are $3^{2 p}$ essentially distinct theta functions of the first order whose characteristic can be formed from rational numbers with denominator 3 . We shall use the $3^{2 p}=81(p=2)$ cubes of these functions, i. e., 81 theta functions of the third order and characteristic zero. Of these only $3^{p}=9$ are linearly independent, so that if such a set of 9 are equated to homogeneous coördinates in a linear space $S_{8}$ they furnish, as the variables (u) change a parametric representation of a manifold $M_{2}$ in $S_{8}$. The order of $M_{2}$ is the number of common zeros of two such functions, i. e., $3^{2} \cdot 2 !=18$.

If we replace $(u)$ by $(u)+(P)$ where $(P)$ is any period then $(\mathrm{K}, \mathrm{p} .371$, $\mathrm{V}, \mathrm{VI})$ the 81 theta cubes all are affected by the same factor of proportionality so that values $(u)^{\prime} \equiv(u)$ furnish the same point on $M_{2}^{18}$. If we replace $(u)$ by $\left(u+\frac{1}{3} P\right)$ then (K, p. 371 , VII, VIII) the 81 theta cubes are permuted cyclically in sets of three to within a factor of proportionality common to all. Hence $(u)^{\prime} \equiv\left(u+\frac{1}{3} P\right)$ is the parametric expression of a collineation of period three which transforms $M_{2}^{18}$ into itself. By allowing $\left(\frac{1}{3} P\right)$ to take all possible values a collineation $G_{81}$ is obtained. If we replace $(u)$ by $-(u)$ then $(\mathrm{K}$, p. $372, \mathrm{IX})$ all the theta cubes, except $[\vartheta(u)]^{3}$ which is unaltered, are interchanged in pairs without extraneous factors. Hence $(u)^{\prime} \equiv-(u)$ is the parametric expression of an involution $I$ which also transforms $M_{2}^{18}$ into itself. These permutations of the functions are independent of variation of the moduli $\tau_{11}, \tau_{12}, \tau_{22}$ of the functions, whence

(46) Any one of a family of $\infty^{3}$ spreads $M_{2}^{13}$ defines the collineation group $G_{2.81}$ under which each member of the family is invariant. The parametric form of $G_{2 \cdot 81}$ is $(u)^{\prime}= \pm(u)+\left(\frac{1}{3} P\right)$. The $G_{2 \cdot 81}$ contains an abelian subgroup $G_{81}$ of signature $(3,3,3,3)$ and also contains 81 involutions conjugate to $I$ under $G_{81}$.

${ }^{*}$ Cf. Krazer, Lehrbuch der Thetafunktionen, pp. 370-72; cited hereafter as $K$. 
We shall speak of $M_{2}^{18}$ as the normal hyperelliptic surface of genus 2 and grade 3 since any surface whose parametric equations contain only functions of the third order and zero characteristic is either $M_{2}^{18}$ or one of its projections.

To obtain a convenient form for $G_{2 \cdot 81}$ let us suppose that a member of the family has been isolated for which $\tau_{12}$ vanishes. The theta functions of the third order and zero characteristic can then be linearly expressed in terms of products of similar elliptic theta functions with respectively variables $u, v$ and moduli $\tau_{11}, \tau_{22}$. If the third periods are $\frac{1}{3} Q$ and $\frac{1}{3} R$ respectively the $G_{2.81}$ is a product of the parametric substitutions

$$
u^{\prime} \equiv e u+\frac{1}{3} Q, \quad v^{\prime} \equiv e v+\frac{1}{3} R(e= \pm 1) .
$$

As we know such linear combinations of the elliptic thetas can be formed that their collineation group $G_{2 \cdot 9}$ is generated by

$$
x_{i}^{\prime}=x_{i+1}, \quad x_{i}^{\prime}=\rho^{i} x_{i}, \quad x_{i}^{\prime}=x_{3-i} \quad(i \equiv 0,1,2 \bmod 3) .
$$

Similar combinations $\overline{x_{i}}$ in the variables $v$ can be formed and the 9 products $x_{i} \overline{x_{j}}$ define special members of the family (46). The $G_{2 \cdot 81}$ of such a special member, and therefore also of the general member, of the family has the generators:

$$
\begin{aligned}
x_{i j}^{\prime}=x_{i+1, j}, \quad x_{i j}^{\prime}=\rho^{i} x_{i j}, \quad x_{i j}^{\prime}=x_{i, j+1}, \\
x_{i j}^{\prime}=\rho^{j} x_{i j}, \quad x_{i, j}^{\prime}=x_{3-i, 3-j} \\
\left(\rho=e^{2 \pi i / 3} ; i, j \equiv 0,1,2 \bmod 3\right) .
\end{aligned}
$$

The set of 81 points $(u) \equiv\left(\frac{1}{3} P\right)$ on $M_{2}^{18}$ is a conjugate set under $G_{81}$. The particular involution $I$ given by $(u)^{\prime} \equiv-(u)$ or $x_{i j}^{\prime}=x_{3-i, 3-j}$ has 16 fixed points on $M_{2}^{18}$ namely the one point $(u) \equiv 0$ included in the above set and the 15 points $(u) \equiv\left(\frac{1}{2} P\right) \neq 0$. All of these points must be found on the fixed spaces of $I$. If in order to bring into evidence these fixed spaces we make the change of coördinates

$$
\begin{aligned}
y_{0} & =x_{00}, & & \\
2 y_{1} & =x_{01}+x_{02}, & & 2 z_{1}=x_{01}-x_{02}, \\
2 y_{2} & =x_{10}+x_{20}, & & 2 z_{2}=x_{10}-x_{20}, \\
2 y_{3} & =x_{11}+x_{22}, & & 2 z_{3}=x_{11}-x_{22}, \\
2 y_{4} & =x_{12}+x_{21}, & & 2 z_{4}=x_{12}-x_{21},
\end{aligned}
$$

we find that the spaces of fixed points of $I$ are an $S_{3}$ with coördinates $z$ determined by $y=0$, and an $S_{4}$ with coördinates $y$ determined by $z=0$. Since in the degenerate elliptic case when $u \equiv 0, x_{0}=x_{1}+x_{2}=0$, and when $u \equiv \frac{1}{2} Q, x_{1}-x_{2}=0$ we see that in general the fixed $S_{4}$ contains the point $(u) \equiv 0$ and the 9 points $(u) \equiv\left(\frac{1}{2} P\right)$ where $\left(\frac{1}{2} P\right)$ has an even characteristic while the fixed $S_{3}$ contains the 6 points $(u) \equiv\left(\frac{1}{2} P\right)$ where $\left(\frac{1}{2} P\right)$ has an odd characteristic. Since a pair of corresponding points of $I$ is projected from either fixed space into a single point of the other fixed space we have 
(49) Each $M_{2}^{18}$ of the family invariant under the $G_{2 \cdot 81}$ generated by (47) has a point $(u) \equiv 0$ in the fixed $S_{4}$ of the involution $I$ of $G_{2 \cdot 81}$. This fixed $S_{4}$ meets $M_{2}^{18}$ further in the 9 even half period points and the fixed $S_{3}$ meets $M_{2}^{18}$ in the 6 odd half-period points. The family of $\infty^{3} M_{2}^{18} s$ is projected from the $S_{3}$ into a family of doubly covered spreads $N_{2}^{6}$ in $S_{4}$ and is projected from the $S_{4}$ into a family of doubly covered surfaces $W_{2}^{4}$ in $S_{3}$.

The general quadratic form in $S_{8}$ contains 9 squares and 36 product terms. These all are theta functions of order 6 and characteristic 0 so that there must be at least 9 relations among them which we find as follows. Assume any relation among the 45 terms. Being an identity it must be transformed into an identity by the collineation $x_{i j}^{\prime}=\rho^{j} x_{i j}$ and we obtain thus three identities. If these be multiplied respectively by $1,1,1$, then by $1, \rho, \rho^{2}$, then by $1, \rho^{2}, \rho$ and in each case added there will be obtained three identities each consisting of those terms of the original identity for which the sum of the second subscripts is a constant mod 3 . Proceeding in the same way with $x_{i j}^{\prime}=\rho^{i} x_{i j}$ we get from each of the three identities three new identities such that in each term the sum of the first subscripts is a constant mod 3 . Hence there must be an identity of one of the 9 following forms and beginning with any one the remaining ones are obtained from it by the collineations $x_{i, j}^{\prime}=x_{i+1, j}, x_{i, j}^{\prime}=x_{i, j+1}$.

$$
\begin{aligned}
& \alpha_{0} x_{00}^{2}+2 \alpha_{1} x_{01} x_{02}+2 \alpha_{2} x_{10} x_{20}+2 \alpha_{3} x_{11} x_{22}+2 \alpha_{4} x_{12} x_{21}=0, \\
& \alpha_{0} x_{01}^{2}+2 \alpha_{1} x_{02} x_{00}+2 \alpha_{2} x_{11} x_{21}+2 \alpha_{3} x_{12} x_{20}+2 \alpha_{4} x_{10} x_{22}=0, \\
& \alpha_{0} x_{02}^{2}+2 \alpha_{1} x_{00} x_{01}+2 \alpha_{2} x_{12} x_{22}+2 \alpha_{3} x_{10} x_{21}+2 \alpha_{4} x_{11} x_{20}=0
\end{aligned}
$$

These are the 9 expected relations with coefficients $\alpha_{0}, \cdots, \alpha_{4}$ which are modular forms still to be determined.

If we apply to these quadrics the involution $I$ the first is unaltered while the others are permuted in pairs. If we add and subtract the members of a pair to form a new pair and then introduce the coördinates $y$ and $z$ from (48) we get the new equations

$$
\begin{aligned}
& \alpha_{0} y_{0}^{2}+2 \alpha_{1} y_{1}^{2}+2 \alpha_{2} y_{2}^{2}+2 \alpha_{3} y_{3}^{2}+2 \alpha_{4} y_{4}^{2} \\
& -2 \alpha_{1} z_{1}^{2}-2 \alpha_{2} z_{2}^{2}-2 \alpha_{3} z_{3}^{2}-2 \alpha_{4} z_{4}^{2}=0, \\
& \alpha_{0} y_{1}^{2}+2 \alpha_{1} y_{0} y_{1}+2 \alpha_{2} y_{3} y_{4}+2 \alpha_{3} y_{2} y_{4}+2 \alpha_{4} y_{2} y_{3} \\
& +\alpha_{0} z_{1}^{2}-2 \alpha_{2} z_{3} z_{4}-2 \alpha_{3} z_{2} z_{4}-2 \alpha_{4} z_{2} z_{3}=0, \\
& \alpha_{0} y_{2}^{2}+2 \alpha_{1} y_{3} y_{4}+2 \alpha_{2} y_{0} y_{2}+2 \alpha_{3} y_{1} y_{4}+2 \alpha_{4} y_{3} y_{1} \\
& +\alpha_{0} z_{2}^{2}+2 \alpha_{1} z_{3} z_{4}+2 \alpha_{3} z_{1} z_{4}-2 \alpha_{4} z_{3} z_{1}=0 \text {, }
\end{aligned}
$$




$$
\begin{gathered}
\alpha_{0} y_{3}^{2}+2 \alpha_{1} y_{4} y_{2}+2 \alpha_{2} y_{4} y_{1}+2 \alpha_{3} y_{0} y_{3}+2 \alpha_{4} y_{2} y_{1} \\
+\alpha_{0} z_{3}^{2}+2 \alpha_{1} z_{4} z_{2}-2 \alpha_{2} z_{4} z_{1}+2 \alpha_{4} z_{2} z_{1}=0, \\
\alpha_{0} y_{4}^{2}+2 \alpha_{1} y_{2} y_{3}+2 \alpha_{2} y_{3} y_{1}+2 \alpha_{3} y_{2} y_{1}+2 \alpha_{4} y_{0} y_{4} \\
+\alpha_{0} z_{4}^{2}+2 \alpha_{1} z_{2} z_{3}+2 \alpha_{2} z_{3} z_{1}-2 \alpha_{3} z_{2} z_{1}=0, \\
z_{1} \pi_{01}+z_{2} \pi_{43}+z_{3} \pi_{24}+z_{4} \pi_{32}=0, \\
z_{1} \pi_{43}+z_{2} \pi_{02}+z_{3} \pi_{14}+z_{4} \pi_{13}=0, \quad\left(\pi_{i k}=\alpha_{i} y_{k}-\alpha_{k}\right. \\
z_{1} \pi_{24}+z_{2} \pi_{14}+z_{3} \pi_{03}+z_{4} \pi_{12}=0, \\
z_{1} \pi_{32}+z_{2} \pi_{13}+z_{3} \pi_{12}+z_{4} \pi_{04}=0,
\end{gathered}
$$

(52) The normal surface $M_{2}^{18}$ is the complete intersection of the nine quadric spreads of (50) or (51).

That it is the complete intersection will follow later from the fact that the projections are completely defined by the above equations.

Since for $(u) \equiv 0$ we have a point on the fixed $S_{4}$ of $I$ the coördinates $z$ of this point are zero and from the simplified form of the first five quadrics (51) we see that the point $(u) \equiv 0$ of $M_{2}^{18}$ is on the hessian $J_{10}$ and the modular forms $\alpha_{0}, \cdots, \alpha_{4}$ are the coördinates of the corresponding point of the steinerian of the quartic spread $J_{4}=y_{0}^{4}+8 y_{0}\left(y_{1}^{3}+\cdots+y_{4}^{3}\right)+48 y_{1} y_{2} y_{3} y_{4}$ in the fixed $S_{4}$ of $I$. However we shall see later that this is only a partial statement of the hessian and steinerian relation. For a general point $y, z$ on $M_{2}^{18}$ the last four equations (51) hold and the $z$ 's are not zero, so that the $y$ 's which are also the coördinates in $S_{4}$ of a projected point pair of $M_{2}^{18}$ must satisfy the equation

$$
K=\left|\begin{array}{llll}
\pi_{01} & \pi_{43} & \pi_{24} & \pi_{32} \\
\pi_{43} & \pi_{02} & \pi_{14} & \pi_{13} \\
\pi_{24} & \pi_{14} & \pi_{03} & \pi_{12} \\
\pi_{32} & \pi_{13} & \pi_{12} & \pi_{04}
\end{array}\right|=0
$$

Hence the doubly covered surface $N_{2}^{6}$ lies on this quartic cone $K$. In order to determine the point $\alpha$ and thereby to locate this cone we observe that for the 6 points of $M_{2}^{18}$ on $S_{3}$ the coördinates $y$ are zero and therefore these points will lie on the five quadrics in $S_{3}$ obtained by setting $y=0$ in (51). If these be multiplied respectively by $\alpha_{0}, 2 \alpha_{1}, \cdots, 2 \alpha_{4}$ and added the result is zero so that the quadrics

$$
\begin{aligned}
\alpha_{0} z_{1}^{2}-2 \alpha_{2} z_{3} z_{4}-2 \alpha_{3} z_{2} z_{4}-2 \alpha_{4} z_{3} z_{2} & =0, \\
\alpha_{0} z_{2}^{2}+2 \alpha_{1} z_{3} z_{4}+2 \alpha_{3} z_{4} z_{1}-2 \alpha_{4} z_{1} z_{3} & =0, \\
\alpha_{0} z_{3}^{2}+2 \alpha_{1} z_{4} z_{2}-2 \alpha_{2} z_{1} z_{4} & +2 \alpha_{4} z_{2} z_{1}=0, \\
\alpha_{0} z_{4}^{2}+2 \alpha_{1} z_{2} z_{3}+2 \alpha_{2} z_{3} z_{1}-2 \alpha_{3} z_{1} z_{2} & =0,
\end{aligned}
$$


meet in the six points, say the $P_{6}^{3}$, in which $M_{2}^{18}$ cuts $S_{3}$. For any one of these points $z$, equations (54) determine the ratios of the $\alpha$ 's to be

$$
\begin{aligned}
& \alpha_{0}=6 z_{1} z_{2} z_{3} z_{4}, \\
& \alpha_{1}=-z_{1}\left(z_{2}^{3}+z_{3}^{3}+z_{4}^{3}\right), \\
& \alpha_{2}=z_{2}\left(z_{1}^{3}+z_{3}^{3}-z_{4}^{3}\right), \\
& \alpha_{3}=z_{3}\left(z_{1}^{3}-z_{2}^{3}+z_{4}^{3}\right), \\
& \alpha_{4}=z_{4}\left(z_{1}^{3}+z_{2}^{3}-z_{3}^{3}\right) . *
\end{aligned}
$$

Now as the moduli vary the $P_{6}^{3}$ of $M_{2}^{18}$ on $S_{3}$ runs over the $S_{3}$ and equations (55) constitute the map of $P_{6}^{3}$ 's in $S_{3}$ upon a certain spread in the fixed $S_{4}$. The mapping is effected by a linear system of $\infty^{4}$ quartic spreads on a Witting configuration, say a $W_{40}$, made up of the 40 points

$$
\begin{aligned}
& z_{i}=1, \quad z_{j}=0 \quad(i \neq j ; i, j=1, \cdots, 4) ; \\
& z_{1}=0, \quad z_{2}^{3}=z_{3}^{3}=z_{4}^{3} ; \\
& z_{2}=0, \bullet \quad z_{3}^{3}=-z_{4}^{3}=z_{1}^{3} ; \\
& z_{3}=0, \quad z_{4}^{3}=z_{1}^{3}=-z_{2}^{3} ; \\
& z_{4}=0, \quad z_{1}^{3}=z_{2}^{3}=-z_{3}^{3} .
\end{aligned}
$$

Three of these quartic surfaces meet in 64 points, of which only 24 are variable, but these divide into $4 P_{6}^{3}$ 's so that a line in $S_{4}$ meets the map of $S_{3}$ under (55) in four points. Since this map must be invariant under the modular group it must be the spread $J_{4}$ and it is easy to verify directly that $J_{4}(\alpha)$ vanishes for the values $\alpha$ given in (55). $\dagger$

Another interesting fact concerning the system of quartic surfaces on $W_{40}$ arises by considering the jacobian of the four quadrics (54), which is a Weddle

${ }^{*}$ Cf. BIII, p. 337; the formulas there given are wrong, since as they stand $J_{4}(Y) \neq 0$. The $Y_{0}$ and $Y_{1}$ should be changed in sign.

$\dagger$ The modular groups in $y$ and $z$ are fully discussed by Klein, Witting, and Burkhardt. It is clear that the $G_{2 \cdot 81}$ contains $40 G_{3}$ 's and that further collineations exist which permute these $G_{3}$ 's. Thus $G_{2 \cdot 81}$ is an invariant subgroup of a collineation $G_{2 \cdot 81 \cdot m}$. The elements of this group must leave the family of $M_{2}^{18}$ 's invariant but must permute its members and therefore must arise by adjoining transformations on $\tau_{i k}$. These additional collineations could be determined readily by finding the collineations on the $z$ 's which permute the points of $W_{40}$, by finding from (55) the corresponding collineations on the $\alpha$ 's which are cogredient with the $y$ 's, and by adjusting the factors of proportionality so that the system (51) in $y, z, \alpha$ is invariant. The modular groups in $y$ alone or $z$ alone are obtained from $G_{2 \cdot 81 \cdot m}$ by considering the subgroup which leaves. $I$ unaltered, a subgroup which is isomorphic with the factor group of $G_{2 \cdot 81}$ under $G_{2 \cdot 81 \cdot m}$. 
quartic with nodes at the $P_{6}^{3}$ common to the four. This jacobian is

$$
\begin{aligned}
& J \equiv \frac{6}{\alpha_{0}}\left|\begin{array}{cccc}
\alpha_{0} z_{1} & -\alpha_{3} z_{4}-\alpha_{4} z_{3}-\alpha_{4} z_{2}-\alpha_{2} z_{4}-\alpha_{2} z_{3}-\alpha_{3} z_{2} \\
\alpha_{3} z_{4}-\alpha_{4} z_{3} & \alpha_{0} z_{2} & \alpha_{1} z_{4}-\alpha_{4} z_{1} & \alpha_{1} z_{3}+\alpha_{3} z_{1} \\
-\alpha_{2} z_{4}+\alpha_{4} z_{2} & \alpha_{1} z_{4}+\alpha_{4} z_{1} & \alpha_{0} z_{3} & \alpha_{1} z_{2}-\alpha_{2} z_{1} \\
\alpha_{2} z_{3}-\alpha_{3} z_{2} & \alpha_{1} z_{3}-\alpha_{3} z_{1} & \alpha_{1} z_{2}+\alpha_{2} z_{1} & \alpha_{0} z_{4}
\end{array}\right|, \\
& J=\left[\alpha_{0}^{3}+2\left(\alpha_{1}^{3}+\cdots+\alpha_{4}^{3}\right)\right] 6 z_{1} z_{2} z_{3} z_{4} \\
& +6\left(\alpha_{0} \alpha_{1}^{2}+2 \alpha_{2} \alpha_{3} \alpha_{4}\right)\left[-z_{1}\left(z_{2}^{3}+z_{3}^{3}+z_{4}^{3}\right)\right] \\
& +\cdots+6\left(\alpha_{0} \alpha_{4}^{2}+2 \alpha_{1} \alpha_{2} \alpha_{3}\right)\left[z_{4}\left(z_{1}^{3}+z_{2}^{3}-z_{3}^{3}\right)\right. \text {. }
\end{aligned}
$$

If then we write $J_{4}$ in symbolic form

$$
J_{4}=(a y)^{4}=(b y)^{4}=\cdots,
$$

we have the simple equation for $J$

$$
J=(a \alpha)^{3}\left(a \alpha^{\prime}\right),
$$

where $\alpha^{\prime}$ can be expressed as a quartic in $z$ by means of (55).

(60) The fixed ' $S_{3}$ of $I$ is mapped in (55) upon the fixed $S_{4}$ by quartic spreads on $W_{40}$. The sets $P_{6}^{3}$ cut out on $S_{3}$ by the $\infty^{3} M_{2}^{18} s$ map upon single points of the quartic spread $J_{4}$ in $S_{4}$ which has the peculiarity of being its own steinerian. If $P_{6}^{3}$ maps upon $\alpha$ on $J_{4}$ the tangent $S_{3}$ to $J_{4}$ at $\alpha$ cuts $J_{4}$ in the map of a Weddle quartic in $S_{3}$ with nodes at $P_{6}^{3}$ and in the linear system on $W_{40}$. Thus the linear system contains $\infty^{3}$ Weddle surfaces one with a node at each point of $S_{3}$.

The following useful identity can be verified easily:

$$
\begin{aligned}
(a \alpha)^{4} \cdot(a y)^{4}-4(a \alpha)^{3}(a y) \cdot(a \alpha) & (a y)^{3} \\
& +3\left[(a \alpha)^{2}(a y)^{2}\right]^{2}=48 K ; \quad \text { i. e., }
\end{aligned}
$$

(62) The locus of lines in $S_{4}$ which cut the quartic spread $J_{4}$ in four self-apolar points is the quartic complex $K$.

We have already noted that the doubly covered $N_{2}^{6}$ lies on the cone $K$ for given $\alpha$ on $J_{4}$. If we multiply the first five quadrics (51) by $\alpha_{0}, 2 \alpha_{1}, \cdots, 2 \alpha_{4}$ respectively and add, the $z$ 's disappear and we have $(a \alpha)^{2}(a y)^{2}=0$. Hence $N_{2}^{6}$ lies on the polar quadric of $\alpha$ as to $J_{4}$. This meets $K$ in an octavic 2-way. But from $(61)$ if $(a \alpha)^{4}=0,(a \alpha)^{2}(a y)^{2}=0$, and $K=0$, then either $(a \alpha)^{3}(a y)=0$ or $(a \alpha)(a y)^{3}=0$. The octavic 2-way breaks up into a quadric and $N_{2}^{6}$, which is the complete intersection of $(a \alpha)^{2}(a y)^{2}=0$ and $(a \alpha)(a y)^{3}=0$. If $\pi$ is any plane on $\alpha, \pi$ meets $J_{4}$ in a quartic curve on $\alpha$ and meets $(a \alpha)(a y)^{3}=0$ and $(a \alpha)^{2}(a y)^{2}=0$ in the polar cubic and conic of $\alpha$ as to this curve. The polar curves meet in only four points outside $\alpha$ 
and from (61) the lines from $\alpha$ to these four points are on $K$, whence $N_{2}^{6}$ has a double point $\alpha$ and its cone of projection from $\alpha$ is $K$.

The argument used above to show that $(u) \equiv 0$ furnishes a point of the hessian $J_{10}$ whose steinerian point $\alpha$ is on $J_{4}$ applies equally well to show that any one of the 10 points of $M_{2}^{18}$ on $S_{4}$ lies on $J_{10}$ and that all have the same steinerian point $\alpha$. Hence the polar cubic of $\alpha$ on $J_{4}$ as to $J_{4}$ has 10 nodes on $J_{10}$. But a cubic spread in $S_{4}$ with 10 nodes is a Segre cubic* and its enveloping cone from a point on it is a Kummer cone, i. e., a cone whose section is a Kummer surface. But the enveloping cone of $(a \alpha)(a y)^{3}=0$ from $\alpha$ is

$$
3\left[(a \alpha)^{2}(a y)^{2}\right]^{2}-4(a \alpha)^{3}(a y) \cdot(a \alpha)(a y)^{3}=0,
$$

which, since $(a \alpha)^{4}=0$, reduces to $K=0$. Hence we have shown that

(63) If $\alpha$ is any point on $J_{4}$ the polar cubic of $\alpha$ as to $J_{4}$ is a Segre cubic spread with ten nodes on $J_{10}$ where $M_{2}^{18}$ cuts $S_{4}$, whose enveloping cone from $\alpha$ is the Kummer cone $K$. The family of $\infty^{3}$ doubly covered $N_{2}^{6}$ 's in $S_{4}$ is obtained by taking the complete intersection of the polar cubic and the polar quadric of $\alpha$ as to $J_{4}$ as $\alpha$ runs over $J_{4}$. The $N_{2}^{6}$ determined by $\alpha$ has a node at $\alpha$ and its cone of projection from $\alpha$ is $K$. The family of $\infty^{3}$ doubly covered surfaces $W_{2}^{4}$ in $S_{3}$ is the system of $\infty^{3} W$ eddle surfaces $J$ which lie in the linear system on $W_{40}$.

The last statement can be proved as follows: Let $y, z$ be a general point on $M_{2}^{18}$ for which therefore not all the $y$ 's nor all the $z$ 's can vanish. This point satisfies the last four equations (51) and we should ordinarily expect to be able to solve them for the ratios of the $y$ 's in terms of $\alpha, z$. The matrix of the system is

$$
\left\|\begin{array}{lllll}
-\alpha_{1} z_{1} & \alpha_{0} z_{1} & \alpha_{3} z_{4}-\alpha_{4} z_{3} & \alpha_{4} z_{2}-\alpha_{2} z_{4} & \alpha_{2} z_{3}-\alpha_{3} z_{2} \\
-\alpha_{2} z_{2} & -\alpha_{3} z_{4}-\alpha_{4} z_{3} & \alpha_{0} z_{2} & \alpha_{1} z_{4}+\alpha_{4} z_{1} & \alpha_{1} z_{3}-\alpha_{3} z_{1} \\
-\alpha_{3} z_{3} & -\alpha_{2} z_{4}-\alpha_{4} z_{2} & \alpha_{1} z_{4}-\alpha_{4} z_{1} & \alpha_{0} z_{3} & \alpha_{1} z_{2}+\alpha_{2} z_{1} \\
-\alpha_{4} z_{4} & -\alpha_{2} z_{3}-\alpha_{3} z_{2} & \alpha_{1} z_{3}+\alpha_{3} z_{1} & \alpha_{1} z_{2}-\alpha_{2} z_{1} & \alpha_{0} z_{4}
\end{array}\right\|
$$

But the same system arranged in terms of $z$ has the determinant $K$ in (8) and it is satisfied by $y=\alpha$ whatever be $z$. Since, for the general point $y, z$ of $M_{2}^{18}, y \neq \alpha$ we must have as the solution of (64) $y_{i}=\alpha_{i} f(z)$ where $f(z)$ vanishes for the general point of $M_{2}^{18}$, i. e., for the general point of $W_{2}^{4}$ as well. By comparing the determinant value of $y_{0}$ in (64) with $J$ in (57) we see that

$$
y_{i}=\alpha_{i} \frac{J}{6}
$$

whence $f(z)$ is $J$.

Let us now seek a parametric equation for the manifold $M_{2}^{18}$ which is determined by a point $\alpha$ on $J_{4}$. Let $P(\bar{y}, \bar{z})$ be the general point on $M_{2}^{18}, P(\bar{y})$

* Cf. Bertini, Introduzione alla geometria projettiva degli iperspazi, pp. 176-87. 
its projection on $S_{4}$, and $P(\bar{z})$ its projection on $S_{3}$. If $y$ is an arbitrary point on the cone $K$ determined by $\alpha$, the line $\overline{\alpha y}$ meets the quadric $(a \alpha)^{2}(a y)^{2}=0$ in a single point other than $\alpha$, which is the required point $P(\bar{y})$ on $N_{2}^{6}$. If $\bar{y}=y+\lambda \alpha$ we have on substituting in $(a \alpha)^{2}(a y)^{2}=0$ and using $(a \alpha)^{4}=0$ that $(a \alpha)^{2}(a y)^{2}+2 \lambda(a \alpha)^{3}(a y)=0$, whence

$$
\bar{y}_{i}=2 y_{i}(a \alpha)^{3}(a y)-\alpha_{i} \cdot(a \alpha)^{2}(a y)^{2} .
$$

The $\bar{z}_{1}, \cdots, \bar{z}_{4}$ were determined from 4 linear equations with the symmetric determinant $K$. If then we denote this determinant bordered with variables $w$ hy $K(w)$

$$
K(w)_{K=0}=b_{i k} w_{i} w_{k}=(z w)^{2} .
$$

Hence we have for any point $y$ on $K$ a unique point $z$ whose coördinates are determined to within sign by $K(w)=(z w)^{2}$, or for which the products $z_{i} z_{k}$ are uniquely determined as minor determinants of $K$. For the required point $P(\bar{z})$ we must have $\bar{z}_{i}=\mu z_{i}$ and we have to determine this two-valued function $\mu$. Either $z$ or $\bar{z}$ will with $\bar{y}$ satisfy the last four equations (51) and we merely have to determine $\mu$ so that the first five are (or any one of them is) satisfied. Let us take then the first equation and set $\bar{y}_{i}$ equal to its value in (66) and $\bar{z}_{i}$ equal to $\mu z_{i}$ where $z_{i} z_{k}$ is obtained from (67). If we note that in (61) when $(a \alpha)^{4}=0$ and $K=0$ then

$$
\left[(a \alpha)^{2}(a y)^{2}\right]^{2}=4 / 3(a \alpha)^{3}(a y) \cdot(a \alpha)(a y)^{3}
$$

we find that $\alpha_{0} \bar{y}_{1}^{2}+2 \sum_{4} \alpha_{1} \bar{y}_{1}^{2}$ becomes

$$
\begin{aligned}
& 4(a \alpha)^{3}(a y)\left\{\left(\alpha_{0} y_{0}^{2}+2 \sum_{4} \alpha_{1} y_{1}^{2}\right) \cdot(a \alpha)^{3}(a y)\right. \\
& \left.-\left(\alpha_{0}^{2} y_{0}+2 \sum_{4} \alpha_{1}^{2} y_{1}\right) \cdot(a \alpha)^{2}(a y)^{2}+\frac{1}{3}\left(\alpha_{0}^{3}+2 \sum_{4} \alpha_{1}^{3}\right) \cdot(a \alpha)(a y)^{3}\right\} .
\end{aligned}
$$

On the other hand we find that $\sum_{4}-2 \alpha_{1} \bar{z}_{1}^{2}$ becomes

$$
\begin{aligned}
2 \mu^{2}\left\{\sum_{4} \alpha_{1}\left[\pi_{02} \pi_{03} \pi_{04}-\sum_{3} \pi_{02} \pi_{12}^{2}+2 \pi_{12} \pi_{13} \pi_{14}\right]\right\} \\
=2 \mu^{2}\left\{-4 \alpha_{1} \alpha_{2} \alpha_{3} \alpha_{4} y_{0}^{3}+3 \alpha_{0} y_{0}^{2} \sum_{4} \alpha_{2} \alpha_{3} \alpha_{4} y_{1}-2 \alpha_{0}^{2} y_{0} \sum_{6} \alpha_{1} \alpha_{2} y_{3} y_{4}\right. \\
\quad+2 y_{0} \sum_{12} \alpha_{1}^{3} \alpha_{2} y_{2}^{2}-4 y_{0} \sum_{6} \alpha_{1}^{2} \alpha_{2}^{2} y_{1} y_{2}+\alpha_{0}^{3} \sum_{4} \alpha_{1} y_{2} y_{3} y_{4} \\
\quad-\alpha_{0} \sum_{12} \alpha_{1}^{3} y_{2}^{3}+\alpha_{0} \sum_{12} \alpha_{1}^{2} \alpha_{2} y_{1} y_{2}^{2}-2 \alpha_{1} \alpha_{2} \alpha_{3} \alpha_{4} \sum_{4} y_{1}^{3} \\
\left.\quad+2 \sum_{12} \alpha_{1}^{2} \alpha_{3} \alpha_{4} y_{1}^{2} y_{2}-2 \sum_{12} \alpha_{1}^{3} \alpha_{2} y_{1} y_{3} y_{4}+2 \sum_{4} \alpha_{1}^{4} y_{2} y_{3} y_{4}\right\}
\end{aligned}
$$

If we substitute the values of the polars in the first brace above the coefficient of $4(a \alpha)^{3}(a y)$ turns out to be four times the coefficient of $2 \mu^{2}$ whence

$$
8(a \alpha)^{3}(a y)+\mu^{2}=0 .
$$

(68) If $\alpha$ is any point of $J_{4}$ and if $y$ is any point of the Kummer cone $K$ (or 
any point of the Kummer surface obtained from an $S_{3}$ section of $K$ ) the parametric equation of the normal hyperelliptic surface $M_{2}^{18}$ in $S_{8}$ in terms of the parameter $y$ is furnished by the equations

$$
\begin{aligned}
(v \bar{y}) & =2(v y) \cdot(a \alpha)^{3}(a y)-(v \alpha) \cdot(a \alpha)^{2}(a y)^{2}, \\
(w \bar{z}) & =\sqrt{-8(a \alpha)^{3}(a y) \cdot K(w)} .
\end{aligned}
$$

The moduli $\alpha_{0}: \alpha_{1}: \cdots: \alpha_{4}$ satisfy the single relation $(a \alpha)^{4}=0$; the parameters $y_{0}: \cdots: y_{4}$ satisfy the single relation $K=0$. This parametric equation of $M_{2}^{18}$ is invariant in form when the cogredient variables $\alpha, y, \bar{y}$ and the contragredient variables $v$ are subjected io the operations of the Burkhardt $G_{25920}$ if at the same time the contragredient variables $\bar{z}, w$ are subjected to the corresponding operations of the isomorphic Maschke $G_{51840}$.

- It is to be noted that the above parametric equations are independent of factors of proportionality in the $\alpha$ 's and the $y$ 's but are not independent of such a factor in the coefficients $a$ of $J_{4}$ which is supposed to be taken with the definite numerical coefficients used throughout. [See Footnote, p. 363.]

The foregoing discussion has developed a number of kleinian forms, i. e., forms in the variables from $S_{3}$ and $S_{4}$ which are unaltered when these variables are transformed under the operations of the isomorphic modular groups. We shall denote such a form by $k(i ; j: k ; l)$ if $i$ and $j$ are the order and class in $S_{4}, k$ and $l$ the order and class in $S_{3}$. If the form contains more than one series of cogredient variables this will be indicated by giving in the proper space the orders in the various series. A first kleinian form can be obtained from the identical covariant ( $v y)$ in $S_{4}$ if $y$ is replaced by the cogredient expressions (55) in $z$. It is

$$
k_{1}(0 ; 1: 4 ; 0)=v_{0} \cdot 6 z_{1} z_{2} z_{3} z_{4}+\cdots+v_{4}\left[z_{4}\left(z_{1}^{3}+z_{2}^{3}-z_{3}^{3}\right)\right] .
$$

The equation $k_{1}=0$ determines for given $z$ the corresponding point $\alpha$ on $J_{4}$; for given $v$ it determines a quartic of the system on $W_{40}$ which if $v$ touches $J_{4}$ is the Weddle $J$. If we operate with $k_{1}$ on $J_{4}$ we get the form $k_{2}$ in (57),

$$
k_{2}(3 ; 0: 4 ; 0)=J \text {. }
$$

The $K(w)$ of $(67)$ is another kleinian form

$$
\begin{aligned}
& k_{3}(\widehat{3,3} ; 0: 0 ; 2)=K(w)=-w_{1}^{2}\left\{\left(\alpha_{0}^{3}+2 \alpha_{1}^{3}\right) y_{2} y_{3} y_{4}\right. \\
& \quad-\left(y_{0}^{3}+2 y_{1}^{3}\right) \alpha_{2} \alpha_{3} \alpha_{4}-\left(\alpha_{0}^{2} y_{0}+2 \alpha_{1}^{2} y_{1}\right) \sum_{3} \alpha_{2} y_{3} y_{4} \\
& +\left(\alpha_{0} y_{0}^{2}+2 \alpha_{1} y_{1}^{2}\right) \sum_{3} y_{2} \alpha_{3} \alpha_{4}+y_{0} \alpha_{1}^{2} \sum_{3} \alpha_{2} y_{2}^{2}-\alpha_{0} y_{1}^{2} \sum_{3} \alpha_{2}^{2} y_{2} \\
& +2 \alpha_{0} \alpha_{1} y_{1} \sum_{3} \alpha_{2} y_{2}^{2}-2 \alpha_{1} y_{0} y_{1} \sum_{3} \alpha_{2}^{2} y_{2}+y_{0} y_{1}^{2} \sum_{3} \alpha_{2}^{3} \\
& \left.-\alpha_{0} \alpha_{1}^{2} \sum_{3} y_{2}^{3}\right\}+\cdots
\end{aligned}
$$


where $\widehat{3,3}$ indicates that $k_{3}$ is of degree 3 in the line coördinates $\widehat{\alpha} y$. For $\alpha$ on $J_{4}$ and $y$ on $K, k_{3}=0$ is the equation of the node of the Weddle at the point $z$ which corresponds to $y$. For $\alpha$ on $J_{4}$ and given $w, k_{3}=0$ is the equation of a cubic cone which touches $K$ along a sextic 2-way cone which corresponds to the plane section of the Weddle.

If we operate ( $y$ on $v$ ) with $k_{3}$ upon the invariant $J_{0,4}$ in $S_{4}$ we get

$$
\begin{aligned}
k_{4}(3 ; 1: 0 ; 2)=w_{1}^{2}\left[1 8 \left\{\alpha_{0} \alpha_{1}^{2}+2 \alpha_{2} \alpha_{3}\right.\right. & \left.\alpha_{4}\right\} v_{0} \\
& \left.-3\left\{\alpha_{0}^{3}+2 \sum_{4} \alpha_{1}^{3}\right\} v_{1}\right]+\cdots .
\end{aligned}
$$

If again we operate ( $\alpha$ on $v$ ) on $J_{0,4}$ we get

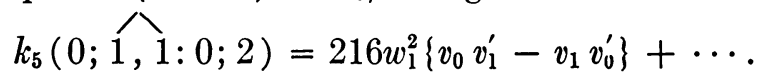

By reason of the self dual character of a collineation group we should expect to find a form $k_{6}\left(\widehat{1,1 ; 0: 2 ; 0)}\right.$ dual to $k_{5}$ and the explicit equation of $k_{6}$ can be found as follows. Multiply the first five quadrics (51) respectively by $y_{0}, 2 y_{1}, \cdots, 2 y_{4}$ and add. Then

$$
\begin{aligned}
(a \alpha)(a y)^{3}+2\left\{\sum_{4} \pi_{01} z_{1}^{2}+2\right. & \left(\pi_{12} z_{3} z_{4}+\pi_{13} z_{4} z_{2}+\pi_{14} z_{2} z_{3}\right. \\
& \left.\left.+\pi_{32} z_{1} z_{4}+\pi_{24} z_{1} z_{3}+\pi_{43} z_{1} z_{2}\right)\right\}=0 .
\end{aligned}
$$

Since $y$ is a point on $N_{2}^{6},(a \alpha)(a y)^{3}=0$ and we take $k_{6}$ to be

$$
k_{6}(\widehat{1,1} ; 0: 2 ; 0)=\sum_{4} \pi_{01} z_{1}^{2}+2 \pi_{12} z_{3} z_{4}+\cdots+2 \pi_{43} z_{1} z_{2} .
$$

If $\alpha$ is on $J_{4}$ and $y$ on $N_{2}^{6}$ (or on $K$ since $y$ occurs only in the combinations $\pi_{i k}$ ), $k_{6}=0$ is a quadric on the 6 nodes of $J$ since it was formed from quadrics (54). Also it is a quadric with a node since its discriminant is $K$. The coördinates $\bar{z}$ of the node are obtained from $K(w)$ and according to $(68) \bar{z}$ is the point of $W_{2}^{4}$ which corresponds to $y$ on $N_{2}^{6}$. Hence

(76) If $M_{2}^{18}$ is determined by $\alpha$ on $J_{4}$ and if $y$ and $z$ are corresponding points on the doubly covered projections $N_{2}^{6}$ and $W_{2}^{4}$ then, for given $y, k_{6}=0$ is the quadric with node at $z$ and on the six nodes of $W_{2}^{4}$.

On account of the 2 to 1 isomorphism between the Maschke and the Burkhardt group we cannot expect to find kleinian forms in $y$ and $z$ alone which are linear in $z$. But such forms which are quadratic in $z$ can be found. If for example we operate $\left(v\right.$ on $y$ ) with $k_{4}$ upon $J_{4}$ we get a $k_{7}(6 ; 0: 0 ; 2)$ and the point equation of this quadric in $w$ would be a form of the sort required. If two such forms, quadratic in $z$ with coefficients containing $y$, are derived

Footnote. Parametric equations for the normal elliptic curves of orders $3,4,5$ to $(68)$ are found in Dr. B. I. Miller's dissertation; these Trans a ction s, vol. 17 (1916), p. 259. The coördinates of a point of the curve are expressed in covariant form in terms of rational functions of the parameter of a point on a line and the radical of such a function. In the above equations the point on a line is replaced by the point on a Kummer surface. 
their pencil will contain at least one and in general four quadric cones. One of these cones can be isolated by the solution of an accessory quartic equation and its node will be a "covariant point," i. e., a point $z$ whose coördinates are functions of $y$ and which undergoes the collineations of the Maschke group when the $y$ 's are subjected to the operations of the Burkhardt group. By the use of such a point the form problem of the Burkhardt group can be solved in terms of a solution of the form problem of the Maschke group.*

We shall however attack the Burkhardt form problem directly and in the next paragraph indicate how it can be solved in the special case when $J_{4}=0$. In $\S 7$ this restriction will be removed by using an accessory quartic equation.

\section{Solution of the SPECIAl Burkhardt form PRoblem}

The form problem of the Burkhardt group when $J_{4}=0$ (referred to as the "special" Burkhardt form problem) reads as follows: Given the values of the absolute invariants $\lambda=J_{12} / J_{6}^{2}, \mu=J_{18} / J_{6}^{3}, \nu=J_{10}^{3} / J_{6}^{5}$ to find the ratios of the coördinates $\alpha_{0}, \cdots, \alpha_{4}$ of a point on $J_{4}=0$ for which these invariants take the assigned values. The problem has 25920 solutions since the spreads $J_{12}-\lambda J_{6}^{2}, \cdots, J_{10}^{3}-\nu J_{6}^{5}=0$ meet $J_{4}=0$ in $4 \cdot 12 \cdot 18 \cdot 30$ $=25920$ points.

We shall develop the solution of this problem under the following heads.

$1^{\circ}$. A point $\alpha$ on $J_{4}=0$ determines a binary sextic (projective to the sextic similarly determined at any one of the set of points conjugate to $\alpha$ on $J_{4}$ ) which is the fundamental sextic of the hyperelliptic functions.

$2^{\circ}$. The absolute invariants of this sextic are precisely the given $\lambda, \mu, \nu$. These will be identified with a known system.

$3^{\circ}$. The equation of this sextic will be expressed in terms of these invariants by means of an accessory square root.

$4^{\circ}$. With this explicitly given sextic, the hyperelliptic algebraic relation of genus two is determined. Assuming that the transcendental operations involved in the determination of the periods of a pair of integrals of the first kind have been effected, the coördinates $\alpha_{0}, \cdots, \alpha_{4}$ are expressed by means of certain known series.

$1^{\circ}$. From the equation (51) of the Kummer cone $K$ determined at a point $\alpha$ of $J_{4}$ we see that the $S_{3},(a \alpha)^{3}(a y)=0$, and the quadric, $(a \alpha)^{2}(a y)^{2}=0$, meet in a trope of $K$. The six double lines of $K$ on this trope are on

$$
(a \alpha)(a y)^{3}=0 \text {. }
$$

Hence the cubic, quadratic, and linear polars of $\alpha$ as to $J_{4}$ meet in six lines of a quadric cone in $S_{3}$, the meet of $(a \alpha)^{3}(a y)=0$ and $(a \alpha)^{2}(a y)^{2}=0$ which

* As to the difficulty of the reduction here indicated cf. BIII, p. 339*. The use of kleinian forms to determine covariant points is illustrated in the article: Coble, Reduction of the sextic equation to the Valentiner form problem, $\mathrm{M}$ a t h.e $\mathrm{m}$ a t is c h e A n nale $\mathrm{n}$, vol. 70 (1911), p. 337 . 
touch at $\alpha$. The binary sextic

$$
S=s_{0} s^{6}+6 s_{1} s^{5}+15 s_{2} s^{4}+\cdots+s_{6}
$$

determined by these six lines on the cone is the fundamental sextic associated with $K$ and therefore with the hyperelliptic theta functions used here.

$2^{\circ}$. We have seen that the polar cubic spread $C \equiv(a \alpha)(a y)^{3}=0$, is on $\alpha$ of $J_{4}$ and has ten nodes on $J_{10}$. Through $\alpha$ there will be six lines which lie entirely on $C$, the six lines of $1^{\circ}$. The lines through $\alpha$ which touch $C$ again are the lines of $K$. This spread $C$ can be mapped from an $S_{3}$ by means of quadrics $q_{a}, \cdots, q_{f}$ on five points $p_{1}, \cdots, p_{5}$ of $S_{3}$ where

$$
C \equiv q_{a}^{3}+\cdots+q_{f}^{3}, \quad q_{a}+\cdots+q_{f} \equiv 0 .
$$

If a sixth point $p$ maps on the point $\alpha=\bar{q}_{a}, \cdots, \bar{q}_{f}$ of $C$ then the section of $C$ by the polar quadric $q_{a}^{2} \bar{q}_{a}+\cdots+q_{f}^{2} \bar{q}_{f}=0$ is the map of a Weddle quartic in $S_{3}$ with nodes at $p_{1}, \cdots, p_{5}, p$. The section of $C$ by the polar space $q_{a} \bar{q}_{a}^{2}+\cdots+q_{f} \bar{q}_{f}^{2}=0$ is the map of a quadric cone in $S_{3}$ with node at $p$ and on $p_{1}, \cdots, p_{5}$. A convenient analytic representation for this is found in $\mathrm{C} 1, \S 1$. The quadric cone meets the Weddle in the 5 lines from $p$ to $p_{1}, \cdots, p_{5}$ and in the cubic curve on the six points. These six curves through $p$ map into the six lines of $1^{\circ}$ on $\alpha$ in $S_{4}$ and the directions on the quadric cone at $p$, which are the same as those on the Weddle at $p$, map into directions on $(a \alpha)^{2}(a y)^{2}=(a \alpha)^{3}(a y)=0$ about $\alpha$. Hence the sextic $S$ in $S_{4}$ is projective to the sextic in $S_{3}$ determined on the quadric cone with node at $p$ by the six curves mentioned and therefore to the sextic determined on the cubic curve by the points $p_{1}, \cdots, p_{5}, p$ (or to the sextic $y_{0}, \cdots, y_{4}$, $\infty$ of $\mathrm{C} 1, \S 1$ ). The invariants of this sextic have been determined as symmetric functions of the $q$ 's $(\mathrm{C} 1,(7),(10))$. Thus the problem before us is: Given a spread $C$ which can be linearly transformed into the special form (77), and a point $\alpha$ on it which is transformed into $\bar{q}_{a}, \cdots, \bar{q}_{f},(a)$ to determine those covariants of $C$ which are transformed into the elementary symmetric functions $q_{2}, \cdots, q_{6}$ of the $q$ 's, and $(b)$ to find their values for the particular point $\alpha=q_{a}, \cdots, \bar{q}_{f}$.

$2^{\circ}(a)$. We have first of all $C=3 q_{3}$. We shall denote the discriminant of the polar quadric of $C$ by $H$ and the dual equation of this quadric in variables $r_{a}, \cdots, r_{f}$ by $B$. If then we take account of the supernumerary coördinates

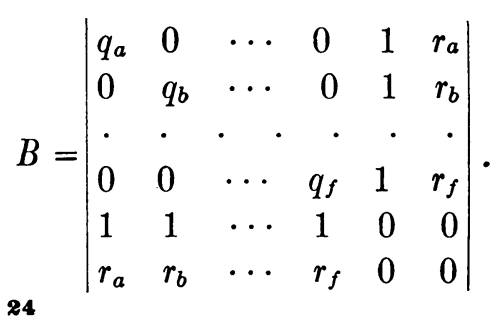


Thus

and

$$
H=-q_{5}
$$

$$
B=\sum_{15} q_{a} q_{b} q_{c} q_{d}\left(r_{e}-r_{f}\right)^{2} .
$$

If we operate with $B$ on $H$ we get

$$
[B, H]=2 \sum_{60} q_{a}^{2} q_{b}^{2} q_{c}^{2} q_{d}=2\left(q_{3} q_{4}-3 q_{2} q_{5}\right) .
$$

If now we allow for a factor $\sigma$ in the coefficients of $C$ we have

$$
\begin{gathered}
C=3 \sigma q_{3}, \quad H=-\sigma^{5} q_{5}, \\
{[B, H]=2 \sigma^{9}\left(q_{3} q_{4}-3 q_{2} q_{5}\right)=2\left(\sigma^{8} q_{4} C / 3+3 H \cdot \sigma^{4} q_{2}\right) .}
\end{gathered}
$$

From the latter syzygy we can determine $\sigma^{8} q_{4}$ and $\sigma^{4} q_{2}$.

If we operate with $B^{2}$ on $H$ we get $-72 q_{6} q_{3}$, whence from

$$
\left[B^{2}, H\right]=-72 \sigma^{13} q_{6} q_{3}=-24 \sigma^{12} q_{6} \cdot C
$$

we can determine $\sigma^{12} q_{6}$.

The term $q_{5}$ derived from $H$ alone does not acquire the proper power of $\sigma$ so it must be modified. The cubic spread in $S_{4}$ has an invariant $A$ of degree 10 in the coefficients which can be obtained by forming according to the Clebsch principle from the invariant $i_{8}$ of the cubic surface in $S_{3}$ a contravariant of $C$ in $S_{4}$ of degree 8 and class 6 and by operating with this contravariant on $C^{2}$. For the above spread the invariant $A$ takes a purely numerical value which we shall denote by $a$. Instead of using $H$ alone we shall use

$$
\frac{1}{\sqrt{a}} \sqrt{A} H=-\sigma^{10} q_{5} \text {. }
$$

$2^{\circ}(b)$. We now identify the cubic spread $C$ with the polar spread $\bar{C}$ of $\alpha$ on $J_{4}$, i. e.,

$$
\begin{aligned}
\bar{C} & =\alpha_{0}\left[y_{0}^{3}+2 \sum_{4} y_{1}^{3}\right]+6 \sum_{4} \alpha_{1}\left(y_{0} y_{1}^{2}+2 y_{2} y_{3} y_{4}\right) \\
& \equiv \sigma\left(q_{a}+\cdots+q_{f}^{3}\right) \equiv C .
\end{aligned}
$$

We calculate the covariants $\bar{H}$ and $\bar{B}$ and the invariant $\bar{A}$ of $\bar{C}$ and equate them to the corresponding forms of $C$ taking account of the determinant $\delta$ of the transformation, and we identify similarly the results of "corresponding operations. We shall want however not the covariants themselves but rather the values which they take at the point $y=\alpha$ so that this substitution can be made after the operations have been completed. Since also we have merely to identify certain numerical coefficients we shall use the special case when $\alpha_{3}=\alpha_{4}=0$ and set

$$
\alpha_{0}^{3}=t_{0}, \quad \alpha_{1}^{3}=t_{1}, \quad \alpha_{2}^{3}=t_{2} .
$$


Then Burkhardt's invariants become

$$
\begin{aligned}
& J_{4}(\alpha)=\alpha_{0}\left[t_{0}+8\left(t_{1}+t_{2}\right)\right], \\
& J_{6}(\alpha)=t_{0}^{2}-20 t_{0}\left(t_{1}+t_{2}\right)-8\left(t_{1}^{2}+t_{2}^{2}\right)+80 t_{1} t_{2}, \\
& J_{10}(\alpha)=\alpha_{0} t_{1} t_{2}\left[-t_{0}+\left(t_{1}+t_{2}\right)\right], \\
& J_{12}(\alpha)=t_{1} t_{2}\left[5 t_{0}^{2}-t_{0}\left(t_{1}+t_{2}\right)-4\left(t_{1}^{2}+t_{2}^{2}\right)+16 t_{1} t_{2}\right], \\
& J_{18}(\alpha)=t_{1}^{2} t_{2}^{2}\left[-t_{0}^{2}+2 t_{0}\left(t_{1}+t_{2}\right)-\left(t_{1}^{2}+t_{2}^{2}\right)+2 t_{1} t_{2}\right] .
\end{aligned}
$$

By a straightforward calculation we find that

$$
\begin{aligned}
\bar{B}= & 16 \cdot 4\left\{4 t_{1} t_{2} \alpha_{0}^{2} u_{0}^{2}-4 t_{1} t_{2} \alpha_{0} u_{0}\left(\alpha_{1} u_{1}+\alpha_{2} u_{2}\right)+t_{2}\left(t_{0}-t_{2}\right) \alpha_{1}^{2} u_{1}^{2}\right. \\
& \left.+t_{1}\left(t_{0}-t_{1}\right) \alpha_{2}^{2} u_{2}^{2}+2 t_{1} t_{2} \alpha_{1} \alpha_{2} u_{1} u_{2}+2 \alpha_{0} \alpha_{1}^{2} \alpha_{2}^{2}\left(t_{0}-t_{1}-t_{2}\right) u_{3} u_{4}\right\} \\
\bar{H}= & 16\left\{-\alpha_{0} \alpha_{1} \alpha_{2} y_{0}^{3}\left(\alpha_{1} y_{2}+\alpha_{2} y_{1}\right)^{2}-y_{0}^{2} \alpha_{0}^{2}\left(\alpha_{1} y_{2}+\alpha_{2} y_{1}\right)^{3}+y_{0}\left[2 t_{2} \alpha_{1}^{2} y_{4}\right.\right. \\
& +\left(4 t_{1}-t_{0}\right) \alpha_{2}^{2} y_{1}^{3} y_{2}+2 \alpha_{1} \alpha_{2}\left(-t_{0}+t_{1}+t_{2}\right) y_{1}^{2} y_{2}^{2}+\alpha_{1}^{2}\left(4 t_{2}-t_{0}\right) y_{1} y_{2}^{3} \\
& \left.+2 \alpha_{2}^{2} t_{1} y_{2}^{4}\right]+2 \alpha_{0} \alpha_{1}^{2} \alpha_{2}^{2} y_{1}^{4} y_{2}+2 \alpha_{0} \alpha_{2}\left(t_{2}+2 t_{1}\right) y_{1}^{3} y_{2}^{2} \\
& +2 \alpha_{0} \alpha_{1}\left(t_{1}+2 t_{2}\right) y_{1}^{2} y_{2}^{3}+2 \alpha_{0} \alpha_{1}^{2} \alpha_{2}^{2} y_{1} y_{2}^{4}+y_{3} y_{4}\left[y_{0}^{3} t_{0} \alpha_{1} \alpha_{2}\right. \\
& +y_{0}^{2} \alpha_{0}\left(t_{0}+2 t_{1}+2 t_{2}\right)\left(\alpha_{1} y_{2}+\alpha_{2} y_{1}\right)+y_{0} \alpha_{0}^{2}\left(t_{0}+2 t_{1}+2 t_{2}\right) y_{1} y_{2} \\
& -4 y_{1}^{3} t_{1} \alpha_{1} \alpha_{2}-2 y_{1}^{2} y_{2} \alpha_{1}^{2}\left(t_{0}+2 t_{1}-4 t_{2}\right)-2 y_{1} y_{2}^{2} \alpha_{2}^{2}\left(t_{0}-4 t_{1}+2 t_{2}\right) \\
& \left.\left.-4 y_{2}^{3} t_{2} \alpha_{1} \alpha_{2}\right]+ \text { terms in } y_{3}^{3} \text { and } y_{4}^{3}\right\} .
\end{aligned}
$$

We now find at once that $\bar{H}_{y=a}=16^{2} J_{10}(\alpha)$, and $\bar{C}_{y=a}=J_{4}(\alpha)$. If we operate with $\bar{B}$ on $\bar{H}$ and set $y=\alpha$ we have

$$
\begin{aligned}
{[\bar{B}, \bar{H}]_{y=a}=} & 16^{3} \alpha_{0} t_{1} t_{2}\left\{2 t_{0}^{3}-6 t_{0}^{2}\left(t_{1}+t_{2}\right)+6 t_{0}\left(t_{1}^{2}+t_{2}^{2}\right)+90 t_{0} t_{1} t_{2}\right. \\
& \left.-2\left(t_{1}^{3}+t_{2}^{3}\right)-30 t_{1} t_{2}\left(t_{1}+t_{2}\right)\right\} \\
= & 16^{2} \cdot 4\left\{J_{4}(\alpha) \cdot J_{12}(\alpha)-3 J_{6}(\alpha) \cdot J_{10}(\alpha)\right\} .
\end{aligned}
$$

If we operate with $\bar{B}^{2}$ on $\bar{H}$ and set $y=\alpha$ we get

$$
\begin{aligned}
{\left[\bar{B}^{2}, \bar{H}\right]_{y=\alpha}=} & 16^{4} \cdot 8 \cdot 3 \alpha_{0} t_{1}^{2} t_{2}^{2}\left\{t_{0}^{3}+6 t_{0}^{2}\left(t_{1}+t_{2}\right)-15 t_{0}\left(t_{1}^{2}+t_{2}^{2}\right)\right. \\
& \left.-34 t_{0} t_{1} t_{2}+8\left(t_{1}^{3}+t_{2}^{3}\right)-8 t_{1} t_{2}\left(t_{1}+t_{2}\right)\right\} \\
= & -16^{4} \cdot 24 J_{4}(\alpha) \cdot J_{18}(\alpha) .
\end{aligned}
$$

The invariant $\bar{A}$ of $\bar{C}$ must be a numerical multiple of $J_{10}(\alpha)$. For its vanishing implies that the polar cubic can not be thrown into the form (77) and this is true only when the hyperelliptic spreads degenerate due to the vanishing of the discriminant of the underlying sextic $S$. But this discriminant is represented on $J_{4}(\alpha)$ by $J_{10}(\alpha) .^{*}$ We shall assume that

$$
\frac{\bar{A}}{a}=\lambda J_{10}(\alpha)
$$

and leave the so defined numerical constant $\lambda$ undetermined.

* Cf. BIII, p. 337 (3) and p. 331 (2). 
We now have after setting $\sigma=1$ in the earlier formulæ the following relations

$$
\begin{aligned}
\bar{C}_{y=a} & =J_{4}(\alpha)=3 q_{3}, \\
\sqrt{\frac{\bar{A}}{a}} \bar{H}_{y=a} & =\sqrt{\lambda J_{10}(\alpha)} \cdot 16^{2} J_{10}(\alpha)=-\delta^{5} q_{5}, \\
\bar{H}_{y=a} & =16^{2} J_{10}(\alpha)=-\delta^{2} q_{5}, \\
{[\bar{B}, \bar{H}]_{y=a} } & =16^{2} \cdot 4\left\{J_{4}(\alpha) \cdot J_{12}(\alpha)-3 J_{6}(\alpha) \cdot J_{10}(\alpha)\right\}=2 \delta^{4}\left(q_{3} q_{4}-3 q_{2} q_{5}\right) \\
{\left[\bar{B}^{2}, \bar{H}\right]_{y=a} } & =-16^{4} \cdot 24 J_{4}(\alpha) \cdot J_{18}(\alpha)=-24 \cdot 3 \delta^{6} q_{3} q_{6} .
\end{aligned}
$$

Hence

$$
\begin{aligned}
& \delta^{2} q_{2}=-2 J_{6}, \\
& \delta^{4} q_{4}=16 \cdot 6 J_{12}, \\
& \delta^{6} q_{6}=16^{4} J_{18}, \\
& \delta^{5} q_{5}=-16^{2} \sqrt{\lambda J_{10}^{3}} .
\end{aligned}
$$

From the two values of $q_{5}$ above we have $\delta=\left(\lambda J_{10}(\alpha)\right)^{1 / 6}$. If we account for it by introducing a factor $1 / \delta$ in the roots of the fundamental sextic $S$ we have finally

(79) The resolvent sextic $\Sigma(\mathrm{C} 1$, p. 317 (9)) of the fundamental sextic $S$ is

$$
Q^{6}-2 J_{6} Q^{4}+16^{2} \cdot 6 J_{12} Q^{2}+16^{2} \sqrt{\lambda J_{10}^{3}} Q+16^{4} J_{18}=0 ;
$$

and the invariants $A, B, C, \Delta$ (notation of $\mathrm{C} 1, \mathrm{p} .317$ (7)) of $S$ are given by the equations

$$
\begin{gathered}
-6 J_{6}=5 A, \quad 16^{2} \cdot 4 \cdot 3^{3} J_{12}=5\left(6 A^{2}-5^{2} B\right), \\
16^{4} \cdot 2 \cdot 3^{4} J_{18}=5\left(-6 A^{3}+3 \cdot 5^{2} A \cdot B+2 \cdot 5^{3} C\right), \\
16^{4} \cdot 3^{5} \lambda J_{10}^{3}=\Delta=\Pi\left(s_{i}-s_{k}\right)^{2}{ }^{*}
\end{gathered}
$$

$3^{\circ}$. In order to exhibit an explicit sextic $S$ we can make use of a typical representation of $S . \dagger$ The sextic has three quadratic covariants connected by an identical relation, $K_{2}=0$, of the second order whose coefficients are rational in $A, B, C, \Delta$. The sextic $S$ is then expressed as a form $S=K_{3}=0$

* I had first attempted to calculate these invariants by the following method. In BIII, p. 331, the sextic $S$ is taken with one root at $\infty$ and the sum of the others zero. Then the coefficients $g_{2}, \cdots, g_{5}$ are given in terms of $[H], \cdots,[\psi]$ which on p. 331 (2) are expressed by means of $\left(f_{12}\right), \cdots,\left(f_{40}\right)$. Then in $\$ 73$, p. 336, the $J_{6}(\alpha)$ and $J_{12}(\alpha)$ for $J_{4}(\alpha)=0$ are calculated in terms of the $\left(f_{12}\right), \cdots,\left(f_{40}\right)$. As expected $J_{6}(\alpha)$ furnished the self apolarity invariant of the sextic $S$ but neither Burkhardt's value [(14), p. 339] of $J_{12}(\alpha)$ nor the different one which I obtained would furnish a second invariant of the sextic. This discrepancy and the one noted above may be due to a single error in the expression for $J_{12}$. An advantage of the method carried through here is that the invariants are identified at once with a known complete system.

† Gordan, Invariantentheorie, p. 302. 
of order three in $l, m, n$ with similar coefficients. Thus the sextic appears as the six points cut out on the conic $K_{2}=0$ by the cubic $K_{3}=0$. In order to introduce a parameter on $K_{2}$ an accessory square root is required. This may be for example the square root of the discriminant of the quadratic in $m, n$ obtained by setting $l=0$ in $K_{2}$. When a point on the conic $K_{2}$ is obtained in terms of a parameter $s$ then the cubic $K_{3}=0$ determines the sextic $S$. Thus we have

If we set

$$
K_{2} \equiv\left|\begin{array}{llll}
A_{l l} & A_{l m} & A_{l n} & l \\
A_{m l} & A_{m m} & A_{m n} & m \\
A_{n l} & A_{n m} & A_{l n} & n \\
l & m & n & 0
\end{array}\right|=a_{11} l^{2}+\cdots+2 a_{23} m n
$$

$$
r=\sqrt{a_{23}^{2}-a_{22} a_{33}}=\sqrt{-2 R^{2} A_{l l}} ; \quad f_{2}=\frac{\partial K_{2}}{\partial m}, \quad f_{3}=\frac{\partial K_{2}}{\partial n},
$$

then we can solve the system of equations

$$
\begin{aligned}
2 R^{2} l & =s, \\
\left(-a_{23}+r\right) f_{2}+a_{22} f_{3} & =a_{22} s^{2}, \\
\left(-a_{23}-r\right) f_{2}+a_{22} f_{3} & =1,
\end{aligned}
$$

for $l, m, n$ as quadratics in $s$, and thereby obtain from $K_{3}$ the sextic $S$. The necessary formulas for explicit expressions in $A, B, C, \Delta$ are given by Gordan, pp. 288-90.

$4^{\circ}$. From the algebraic relation $t=\sqrt{S}$ we assume that the periods $\omega_{i, 1}$, $\omega_{i, 2}(i=1, \cdots, 4)$ of a pair of integrals of the. first kind have been calculated.* From the known series for $X_{a \beta} \dagger$ these quantities are calculated for $(u) \equiv 0$. Then formulæ (48) furnish the coördinates of a point $y$ on $J_{10}$ whose polar quadric as to $J_{4}$ has a double point at the required point $\alpha$ on $J_{4}$.

\section{Solution of the general BuRkhardT Form PROBlem}

We shall state the Burkhardt form problem as follows: Given the numerical values of $J_{4}, J_{6}, J_{10}, J_{12}, J_{18}$ to find the ratios of the coördinates $y$ for which these forms take the given values. If the ratios are found the actual coördinates can be obtained to within sign by using the numerical value of $J_{6} / J_{4}$ to determine the square of the factor of proportionality. The solution required can be given in terms of the solution of the special problem considered in $\S 6$ by conventional methods. $\S$ We shall employ the phrase "de-

* Various methods for this are reviewed in BI, $\S 45$, p. 277. That of Wiltheiss, $\mathrm{M}$ a t h e mat is che Annalen, vol. 31, p. 141, would be more in line with the above account since it implies no separation of the roots of $S$.

$\dagger$ BII, p. 171 (17).

$\ddagger$ Cf. BII, §§ 51-2, p. 214.

$\S$ Cf. Klein, Ikosaeder, II, $5, \S 2$, p. 241. 
terminate polynomial $J_{i}$ " to indicate a polynomial in $J_{4}, J_{6}, \cdots, J_{18}$ with numerical coefficients and of degree $i$ in $y$. These numerical coefficients can be calculated once for all (which we assume has been done in the cases below) and the polynomial takes a numerical value when the form problem is given.

Let $y$ be one of the required points. It can be given by the equation $J_{1,1}=0$. Then $\lambda J_{1,1}+J_{7,1}=0$ is for variable $\lambda$ a line " covariant" with $y$, i. e., the relation between $y$ and the line is unaltered under the transformations of $G_{25920}$ because of the invariance of $J_{1,1}$ and $J_{7,1}$. This line meets $J_{4}$ in four points whose parameters $\lambda$ are the roots of the equation

$$
J_{4} \lambda^{4}+J_{10}^{\prime} \lambda^{3}+J_{16}^{\prime} \lambda^{2}+J_{22}^{\prime} \lambda+J_{28}^{\prime}=0 .
$$

In this equation $J_{i}^{\prime}$ is a determinate polynomial. A root $\lambda$ of (80) is an irrational invariant of $G_{25920}$ of degree 6 . If one such root-an accessory irrationality-has been adjoined, the point $J_{1,1} \lambda+J_{7,1}=0$ is a point covariant with $y$ and on $J_{4}$.

We shall denote by $\bar{J}_{6}, \bar{J}_{10}, \bar{J}_{12}, \bar{J}_{18}$ the values which $J_{6}, \cdots, J_{18}$ take for this covariant point. Then

$$
\bar{J}_{\rho}=J_{\rho} \lambda^{\rho}+J_{\rho+6}^{\prime} \lambda^{\rho-1}+\cdots+J_{\rho+6 k}^{\prime} \lambda^{\rho-k}+\cdots+J_{7_{\rho}}^{\prime},
$$

where $J_{\rho+6 k}^{\prime}$ is a determinate polynomial. Since by means of $(80) J_{4}^{\rho-3} \lambda^{\rho}$ can be expressed linearly in terms of $\lambda^{3}, \lambda^{2}, \lambda, 1$ with determinate polynomial coefficients we can set

$$
J_{4}^{\rho-3} \bar{J}_{\rho}=J_{\rho_{0}} \lambda^{3}+J_{\rho_{1}} \lambda^{2}+J_{\rho_{2}} \lambda+J_{\rho_{3}} \quad(\rho=6,10,12,18),
$$

where $J_{\rho_{i}}$ is a determinate polynomial, for which $\rho_{i}=11 \rho+6 i-30$.

We shall denote by $\bar{J}_{1,1}, \bar{J}_{7,1}, \bar{J}_{9,1}, \bar{J}_{13,1}, \bar{J}_{15,1}$ the values which $J_{1,1}$, $\cdots, J_{15,1}$ take when for $y$ there is substituted the covariant point

on $J_{4}$. Then

$$
\lambda J_{1,1}+J_{7,1}=0
$$

$$
\bar{J}_{\sigma, 1}=J_{\sigma, 1} \lambda^{\sigma}+J_{\sigma+6,1}^{\prime} \lambda^{\sigma-1}+\cdots+J_{\sigma+6 k, 1}^{\prime} \lambda^{\sigma-k}+\cdots+J_{7 \sigma, 1}^{\prime},
$$

where $J_{\sigma+6 k, 1}^{\prime}$ can be expressed linearly in terms of $J_{1,1}, \cdots, J_{15,1}$ with determinate polynomial coefficients. Again multiplying by $J_{4}^{\sigma-3}$ to reduce the exponents of $\lambda$ to 3 or less we can set

$$
\begin{aligned}
J_{4}^{\sigma-3} J_{\sigma, 1}= & {\left[J_{\sigma_{0,1}} \lambda^{3}+J_{\sigma_{1,1}} \lambda^{2}+J_{\sigma_{2,1}} \lambda+J_{\sigma_{3,1}}\right] J_{1,1} } \\
& +\left[J_{\sigma_{0,7}} \lambda^{3}+J_{\sigma_{1,7}} \lambda^{2}+J_{\sigma_{2,7}} \lambda+J_{\sigma_{3,7}}\right] J_{7,1} \\
& \cdot \cdot \cdot \cdot \cdot \cdot \cdot \cdot \cdot \cdot \cdot \cdot \cdot \cdot \cdot \cdot \cdot \cdot \cdot \cdot \cdot \cdot \cdot \cdot \cdot \cdot J_{\left.\sigma_{3,15}\right] J_{15,1},} \cdot(\sigma=1,7,9,13,15), \\
& +\left[J_{\sigma_{0,15}} \lambda^{3}+J_{\sigma_{1,15}} \lambda^{2}+J_{\sigma_{2,15}} \lambda+J\right.
\end{aligned}
$$


where $J_{\sigma_{i j}}$ is a determinate polynomial and $\sigma_{i j}=11 \sigma+6 i-j-30$. All the determinate polynomials that occur above are of even degree so that $J_{45}$ can not appear. Of course if any of the degrees indicated are negative the corresponding terms do not appear.

To solve the given problem we first find from the numerical values of $J_{4}, \cdots, J_{18}$.the numerical values of the determinate polynomials in (80), (81), (82). Equation (80) is then solved and a value of the accessory irrationality $\lambda$ obtained. With this value of $\lambda$ and the given value of $J_{4}$ we find from (81) the values of $\bar{J}_{\rho}$. These are the known quantities in the special Burkhardt form problem and we find as in $\S 6$ the covariant point $\alpha$ on $J_{4}$ such that $(v \alpha)=\lambda J_{1,1}+J_{7,1}$. The coördinates of $\alpha$ can be determined to within sign by the numerical value of $\bar{J}_{12} / \overline{J_{10}}$. With these coördinates $\alpha$ the linear forms $\bar{J}_{\sigma, 1}$ of (82) can be determined to within sign. Since now the left members of (82) are completely known to within a change of sign throughout and the coefficients of $J_{\sigma, 1}$ in the right members also are known we can solve the five linear equations for $J_{1,1}$ and therefore determine to within sign the coefficients $y_{0}, \cdots, y_{4}$ of $v_{0}, \cdots, v_{4}$.

With this the above sketch of the processes involved in the determination of the lines on a cubic surface is complete. In addition to adjoining the square root of the discriminant of the surface we have introduced an accessory square root in $\S 6$ and an accessory quartic irrationality in this paragraph. These are to be compared with the two accessory square roots required by the method of Klein which is based on the form problem of the $z$ 's. The essential difference between the problem of the $y$ 's and the problem of the $z$ 's is that the latter implies an isolation of a root of the fundamental sextic* while the former does not. Much depends also on the way in which the transcendental operations are carried out. If for example we set the problem: Given the numerical values of $J_{6}, \cdots, J_{18}$ when $J_{4}=0$ to find the ratios of the coördinates of the $10 \cdot 25920$ points on $J_{10}$ which are the nodes of the polar cubics of the 25920 points on $J_{4}$; then the algebraic adjunction of a solution of the special form problem would merely reduce the problem to the solution of an equation of degree 10 with a sextic resolvent. But if we proceed to effect the solution of the special form problem as in $\S 6$ by the adjunction of an accessory square root then after the periods of a pair of integrals have been obtained the required points are found by substituting the 10 even half periods (including the zero half period in the transcendental expressions for the $y$ 's). We may note also that the closing remarks in $\$ 5$ indicate that a quartic irrationality may be unavoidable in effecting the solution of the problem of the $y$ 's in terms of the problem of the $z$ 's.

* Cf. BIII, $\S 66,68$, p. 327 . 


\section{Comparison WITH THE QUINTIC EQUATION}

Many of the ideas in this series of articles had their origin in an earlier paper entitled "An application of the form problems associated with certain Cremona groups to the solution of equations of higher degree."* This paper contained a detailed application to the quintic and it may be of interest to trace the striking analogy between the problem of the quintic as there set forth and the problem of the determination of the lines on a cubic surface as presented in this article.

The quintic with ordered roots determined an ordered $P_{5}^{2}$, four of whose points were taken at a base in $S_{2}$, and the coördinates $x, y, u \dagger$ of the remaining point determined in turn the ordered quintic. The permutations of the roots of the quintic led to the operations of a Cremona $G_{5 !}$ in $x, y, u$ whose invariants were the invariants of the quintic itself. This corresponds to the content of our present $\S 1$ except that here the cubic surface determines 72 ordered $P_{6}^{2}$ 's and the Cremona group is the extended group $G_{6,2}$ of order $72 \cdot 6$ ! rather than $G_{6 !}$. The transition from the quintic equation to the solution of the form problem of $G_{5 !}$ was accomplished by a typical representation analogous to the process used in $\S 2$. The simplest linear system of irrational invariants of the quintic of dimension 5 divided under the operations of the invariant even subgroup $G_{151}$ into two skew linear systems of dimension 2 which experienced under $G_{15 !}$ the linear transformations of Klein's contragredient groups of the $A$ 's and of the $A^{\prime \prime}$ 's. Precisely similar facts concerning $\Gamma_{6,2}$ appear in (36) and (40) of $\S 3$. The solution of the form problem of $G_{15 \mathrm{~s}}$ was accomplished by the use of invariants linear in the $A^{\prime \prime}$ s- the same device as is employed in $\S 4$. Here the analogy ends since the solution of the problem of the $A$ 's given in the earlier paper was effected by a special method.

If however we take into account the results of Miss Miller (loc. cit., pp. 278-83) which furnish the analog of $\S 5$, there would seem to be little doubt that a binary quartic could be attached to each point of the conic invariant under the group of the $A$ 's-a quartic projective to that which determines the elliptic quintic in $S_{4}$. Then developments (in which $J_{4}$ is replaced by the invariant conic) precisely parallel to those of $\S \S 6,7$ could be made and the analogy between the two given problems would persist throughout.

BALTIMORE,

July 28, 1916

* Coble, these Tra n s a c t i o n s, vol. 9 (1908), p. 396.

$\dagger$ As a matter of fact the use of a superfluous coördinate was more advantageous in that certain results of Clebsch could be utilized. 